Article

\title{
Synthesis of Chromeno[3,4-b]piperazines by an Enol-Ugi/Reduction/Cyclization Sequence
}

\author{
Ana Bornadiego (D), Ana G. Neo*(D) and Carlos F. Marcos*(D) \\ Laboratory of Bioorganic Chemistry \& Membrane Biophysics (L.O.B.O.), Department of Organic and Inorganic \\ Chemistry, University of Extremadura, 10003 Cáceres, Spain; bornadiegos@unex.es \\ * Correspondence: aneo@unex.es (A.G.N.); cfernan@unex.es (C.F.M.); Tel.: +34-9-2725-7158 (C.F.M.)
}

check for updates

Citation: Bornadiego, A.; Neo, A.G.; Marcos, C.F. Synthesis of Chromeno[3,4- $b]$ piperazines by an Enol-Ugi/Reduction/Cyclization Sequence. Molecules 2021, 26, 1287. https://doi.org/10.3390/ molecules26051287

Academic Editor: Vincenzo Piccialli

Received: 15 February 2021

Accepted: 23 February 2021

Published: 27 February 2021

Publisher's Note: MDPI stays neutral with regard to jurisdictional claims in published maps and institutional affiliations.

Copyright: (c) 2021 by the authors. Licensee MDPI, Basel, Switzerland. This article is an open access article distributed under the terms and conditions of the Creative Commons Attribution (CC BY) license (https:// creativecommons.org/licenses/by/ $4.0 /)$.
Abstract: Keto piperazines and aminocoumarins are privileged building blocks for the construction of geometrically constrained peptides and therefore valuable structures in drug discovery. Combining these two heterocycles provides unique rigid polycyclic peptidomimetics with drug-like properties including many points of diversity that could be modulated to interact with different biological receptors. This work describes an efficient multicomponent approach to condensed chromenopiperazines based on the novel enol-Ugi reaction. Importantly, this strategy involves the first reported post-condensation transformation of an enol-Ugi adduct.

Keywords: multicomponent reactions; isocyanides; peptidomimetics; heterocycles; piperazines; pyrazines; chromones; coumarins; enols; Ugi reaction

\section{Introduction}

Peptidomimetics are molecules structurally related to peptides that can interact at the same receptors of their prototypes [1,2]. They have attracted an enormous medical interest as they present similar, or sometimes opposite, effects to the equivalent peptide, but display more favorable pharmacological properties. Geometrically constrained peptidomimetics are an important class of peptide analogues containing cyclic structures that result in reduced conformational flexibility and usually well-defined secondary structures [3]. This frequently results in enhanced affinities for biological receptors, leading to improved biological activities [4]. In fact, many biologically active natural products present rigid peptide-like motives able to strongly bind to their target biomolecules.

Heterocycles are valuable building blocks to introduce defined structural constrains in peptide sequences [3,4]. Among the most attractive rigid peptidic scaffolds for drug discovery are piperazines [5-10], which are present in many alkaloids and pharmaceuticals that can bind to a wide range of receptors [11]. Additionally, 3-aminocoumarins are considered privileged structures, present in various biologically active natural didepsipeptides, such as bacterial antibiotics novobiocin [12-14], coumermycines [15] and cacibiocins [16], and marine-derived fungal metabolites trichodermamides [17-19].

Conventional methods for the synthesis of these compounds commonly consist in multi-step procedures, including several protection and deprotection reactions. In recent years, more convenient multicomponent approaches have been developed to straightforwardly generate diversely substituted constrained peptidomimetics in one or a few reaction steps. Thus, piperazines and pyrazines have been synthesized by modified Ugi condensations [20] and post-condensation transformations of Ugi [7,21-23] or Ugi-Smiles products $[24,25]$. Different multicomponent strategies have also been used in the synthesis of polycyclic coumarins [26].

Both condensed polycyclic piperazines [27] and coumarins [28] (Figure 1) are unique in terms of structure and properties, reaching larger areas of the chemical space of biologically relevant compounds, as well as a wider diversity of target proteins. Pharmacologically relevant polycyclic pyrazines include praziquantel (1), used as the primary medication for 
human schistosomiasis [29], trabectedin (2), a marine-derived orphan drug that has been approved for the treatment of soft tissue sarcomas [30], and antidepressant mirtazapine (3) [31]. On the other hand, many polycyclic coumarins have distinct biological activities. Notable examples are phytoestrogen coumestrol (4) [32], antioxidant and anticancer ellagic acid (5) [33], antimalarial dioncolactone (6) [34], cannabinoid agonists cannabilactones (7) [35] and neo-tanshinlactone (8), a natural compound known for its specificity and selectivity towards the breast cancer cells [36]. Thus, combining the coumarin and pyrazino scaffolds in the same structure opens new opportunities to develop molecules with novel and selective biological activities [37].<smiles>O=C(C1CCCCC1)N1CC(=O)N2CCc3ccccc3C2C1</smiles>

1 (Praziquantel)

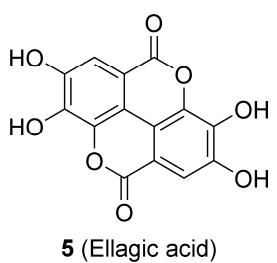

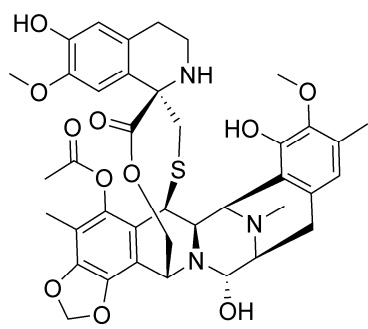

2 (Trabectedin)<smiles>CN1CCN2c3ncccc3Cc3ccccc3C12</smiles>

3 (Mirtazapine)<smiles>COc1cc2c(=O)oc3c4c(ccc3c2c2cccc(O)c12)C[C@@H](C)N[C@H]4C</smiles>

6 (Dioncolactone A)<smiles>O=c1oc2cc(O)ccc2c2oc3cc(O)ccc3c12</smiles>

4 (Coumestrol)<smiles>[R]c1cc(O)c2c(c1)oc(=O)c1ccc(C)cc12</smiles>

7 (Cannabilactone)

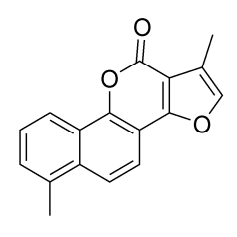

8 (Neo-tanshinlactone)

Figure 1. Some bioactive polycyclic pyrazines and coumarins.

Reports on chromenopyrazines are scarce, but some interesting examples have been described. Thus, chromeno[3,4-b]pyrazines (9) [38] and the structural related pyrazino[2,3c]quinolin-5(6H)-ones (10) [39] are known to have antimicrobial and anticancer activities. Additionally, furochromenopyrazines (11) present similar photobiological activities than psoralens (Figure 2) [40]. Although some syntheses of pyrazino[2,3-c]quinolin-5(6H)ones have been reported [41,42], literature review reveals a limited number of known synthesis of chromenopyrazines (Scheme 1) [43,44]. For example, Pal and co-workers used an $\mathrm{AlCl}_{3}$-induced $\mathrm{C}-\mathrm{C}$ bond formation followed by transition metal mediated couplingcyclization to synthesize chromeno[4,3-b]quinoxalines [43]. More recently, the group of Balci reported a synthesis of chromenopyrazinone derivatives in four reaction steps, starting from salicylaldehyde [44]. These syntheses require the use of environmentally noxious transition metals and permit a limited diversity of products. Consequently, the development of simple and efficient synthetic methods is indispensable to further explore the chromenopyrazine structure.<smiles>O=c1oc2ccc([Tl])cc2c2c1Nc1cc(F)c(P)cc1N2</smiles>

(9)<smiles>COc1ccc2[nH]c(=O)c(=O)[nH]c2c1</smiles>

(10)<smiles>O=c1oc2cc3occc3cc2c2nccnc12</smiles>

(11)

Figure 2. Some bioactive chromenopyrazines and quinolinopyrazines. 
Pal's work:

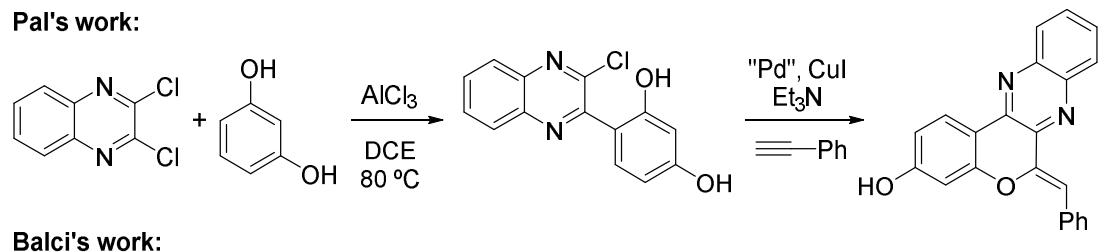

Balci's work:

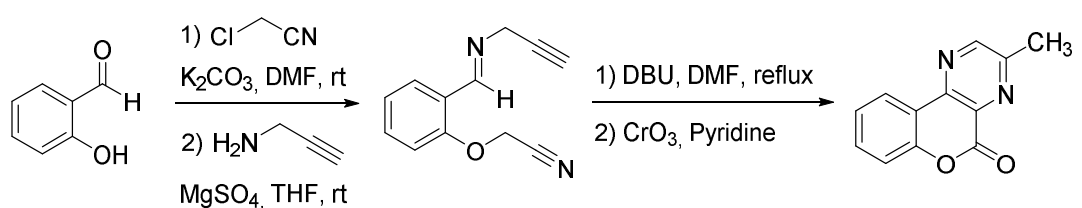

This work:

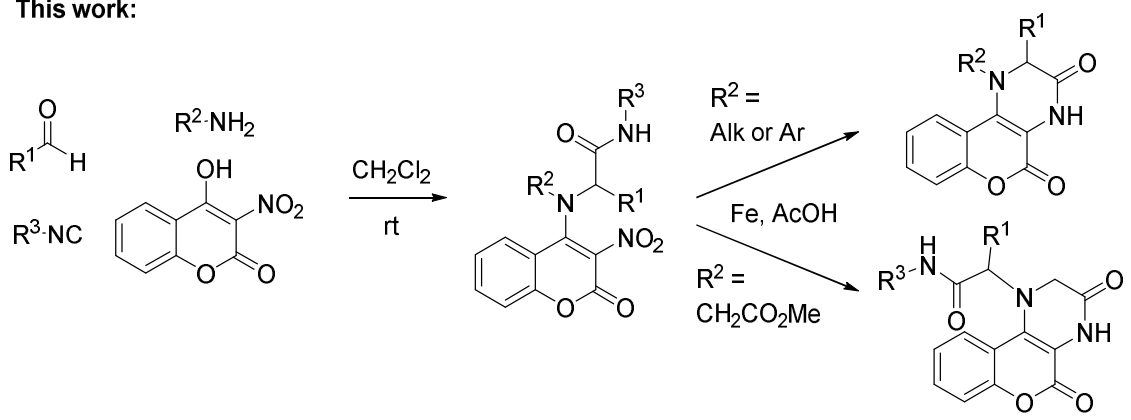

Scheme 1. Representative syntheses of chromenopyrazines.

We have previously used multicomponent reactions of isocyanides for the synthesis of peptide and pseudopeptide derivatives [45-48]. As part of this research line, here we report the multicomponent synthesis of chromeno[3,4-b]pyrazin-5-ones as geometrically restricted peptidomimetics.

\section{Results and Discussion}

We have recently reported the effective multicomponent enol-Ugi condensation of enols (15), aldehydes (12), amines (13) and isocyanides (14) leading to polysubstituted heterocyclic enamines (17; Scheme 2) [49-51]. The enol-Ugi condensation of 4-hydroxy3-nitro-coumarin (15) and cyclohexyl isocyanide (14a) with different amines (13a-d) and aldehydes $(\mathbf{1 2 a}-\mathbf{e})$ or the corresponding preformed imines $(\mathbf{1 6 a}-\mathbf{k})$ leads to aminoacylcoumarins (17a-k) in good to excellent yields (Table 1).<smiles>[R]C=CC</smiles>

(12)

$\mathrm{R}^{3} \cdot \mathrm{NC}$

(14)<smiles></smiles>

(15)<smiles>[R3]NC(=O)C([R])N([R])c1c(F)c(=O)oc2ccccc12</smiles>

(17)

\section{$\mathrm{E}=$ electron-withdrawing group}

Scheme 2. Enol-Ugi condensation of 4-hydroxycoumarin. 
Table 1. Synthesis of enol-Ugi adducts 17 and chromenopyrazines $19^{\mathrm{a}, \mathrm{b}, \mathrm{d}}$.

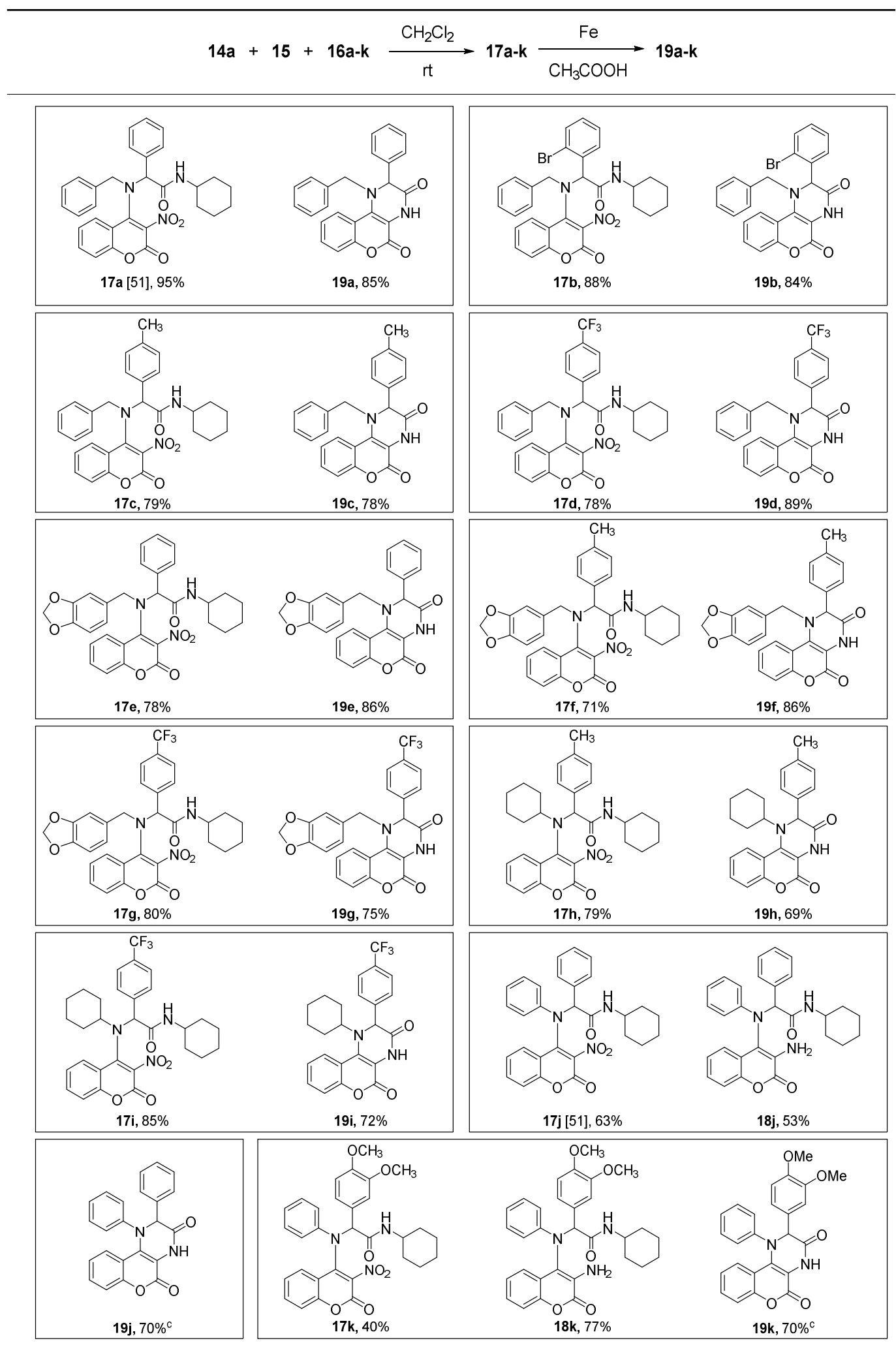

a Procedure for the synthesis of 17a-k: Isocyanide 14 (1 equiv) and enol 15 (1 equiv) were added to a solution of imine 16 (1 equiv) in $\mathrm{CH}_{2} \mathrm{Cl}_{2}$ and stirred at $20^{\circ} \mathrm{C}$ for $3 \mathrm{~h}$. ${ }^{\mathrm{b}}$ Procedure for the synthesis of 19a-i and 18j,k: Enol-Ugi adduct 17 ( 1 equiv) and iron powder ( 24 equiv) were stirred at $\mathrm{rt}$ for $2-4 \mathrm{~h}$. ${ }^{\mathrm{c}}$ The reaction was performed at $150{ }^{\circ} \mathrm{C} .{ }^{\mathrm{d}}$ All yields refer to isolated yields. 
Reduction of the nitro group in the enol-Ugi adducts (17a-k) with iron in acetic acid takes place smoothly at room temperature to afford amino intermediates (18a-k) that are usually not isolated. In the case of enol-Ugi adducts derived from aliphatic amines $(\mathbf{1 7} \mathbf{a}-\mathbf{i})$ the spontaneous intramolecular attack of the amine on the amide group generates a pyrazine ring (19a-i) with loss of cyclohexylamine (Scheme 3). Conversely, enol-Ugi adducts derived from aromatic amines $(\mathbf{1 7} \mathbf{j}, \mathbf{k})$ give stable aminocoumarins $(\mathbf{1 8 j}, \mathbf{k})$ that can be isolated. However, when the reduction/cyclization was carried out at $150{ }^{\circ} \mathrm{C}$ the corresponding chromenopyrazines $(\mathbf{1 9 j}, \mathbf{k})$ were directly obtained (Table 1$)$.<smiles>[R]NC(=O)C([R])N([R])c1c([N+](=O)[O-])c(=O)oc2ccccc12</smiles>

(17)

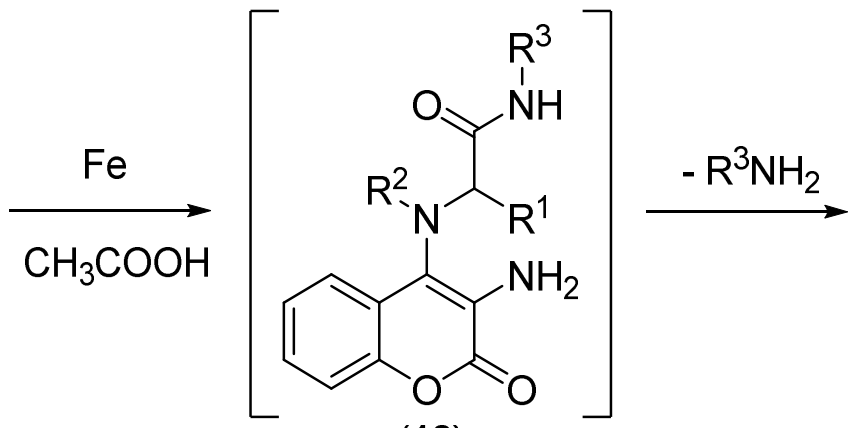

(18)

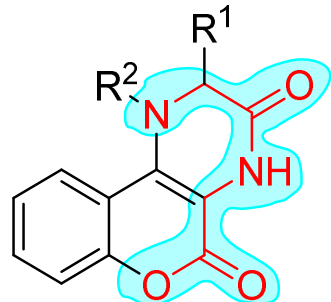

(19)

Scheme 3. Postcondensation synthesis of chromenopyrazinones. Dipeptidic structure is shaded in light blue colour.

A rigid dipeptidic structure is comprised in the pyrazine and pyranone rings of chromenopyrazines (19; Scheme 3). In order to extend the peptidic skeleton of these geometrically constrained dipeptides, we decided to use esters of amino acids as amino components of the enol-Ugi reaction (Scheme 4). Accordingly, the four-component reaction of 4-hydroxy-3-nitro-coumarin (15), different isocyanides (14a-d) and aldehydes (12a,c,d) with glycine methyl ester (13e) gave the corresponding adducts (17l-r) in good yields. The analogous condensation of $\beta$-alanine (13f) similarly gave adduct 17s (Table 2).

$\mathrm{R}^{1} \stackrel{\mathrm{O}}{\mathrm{H}}$

(12)<smiles>[R9]NCc1ccc2oc(=O)c([N+](=O)[O-])c(O)c2c1</smiles>

(15)<smiles>[R]NC(=O)C([R])N(c1c([N+](=O)[O-])c(=O)oc2ccccc12)C([R])C(=O)OC</smiles>

(17)

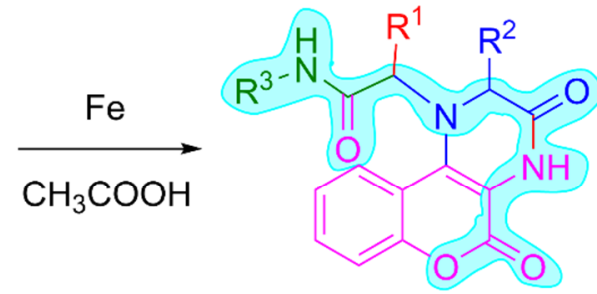

(20)

Scheme 4. Synthesis of chromenopyrazine tripeptides. Tripeptidic structure is shaded in light blue colour.

Interestingly, in this case, reduction of the nitro group does not lead to cyclization by the attack on the amide, as with adducts $\mathbf{1 7} \mathbf{a}-\mathbf{k}$. The attack of the amine occurs instead on the more reactive ester group derived from the glycine methyl ester, affording pyrazines 201-r (Scheme 4; Table 2). The amide group brought by the isocyanide component is thus preserved in the product delivering a new element of diversity, as different isocyanides can be used (Table 2). Rigid retropeptidic tripeptides are obtained in this manner. The peptide sequence could theoretically be grown from the isocyanide-derived amide to obtain peptides with an inverted rigid $N$-terminus. 
Table 2. Synthesis of enol-Ugi adducts of amino acids (17) and chromenopyrazines 19 and 20 a,b,c .

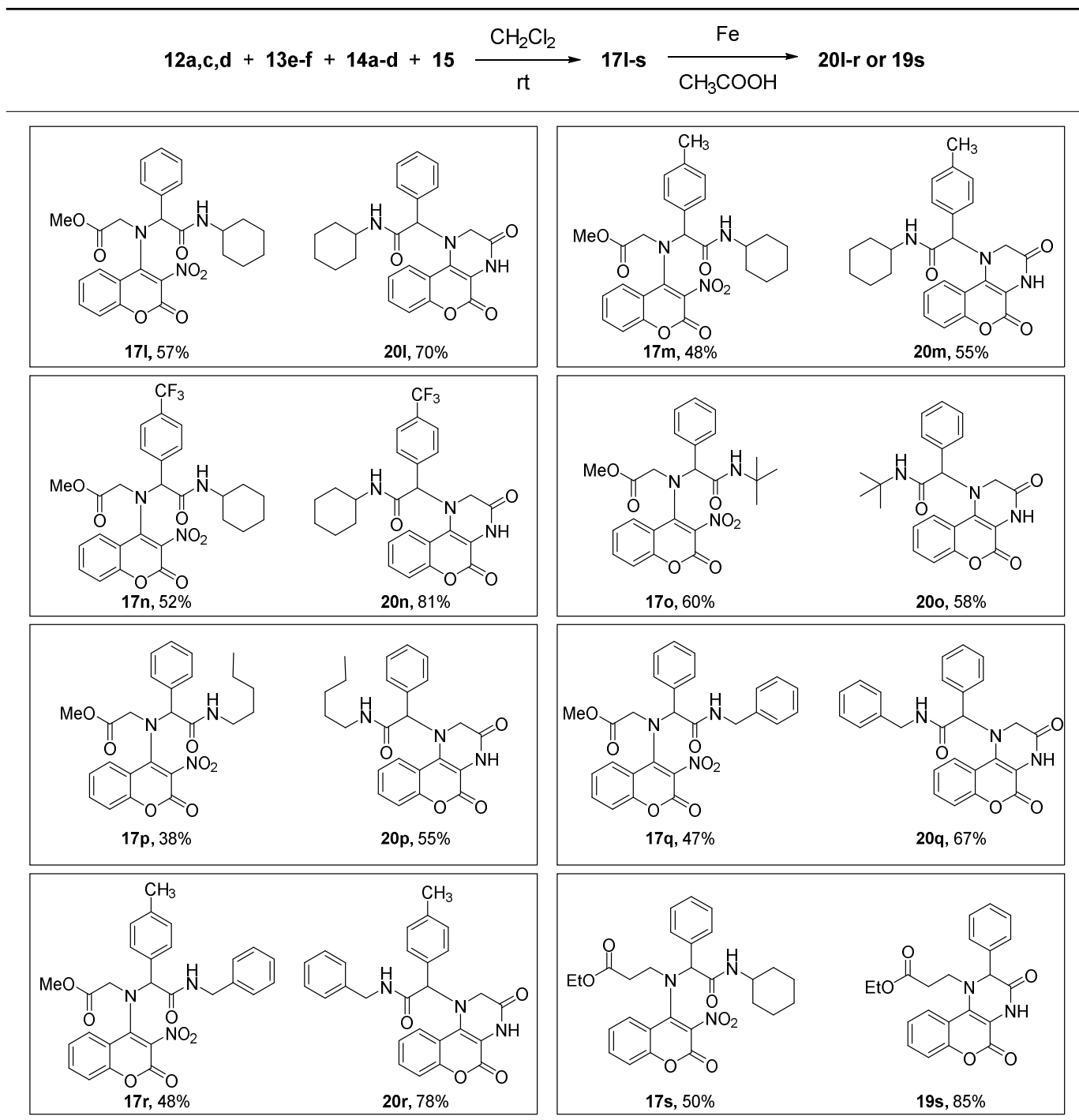

a Procedure for the synthesis of 17l-s: A solution of amine 13 (1 equiv) and aldehyde 12 (1 equiv) in of dry acetonitrile was stirred for $15 \mathrm{~min}$ at rt; isocyanide 14 ( 1 equiv) and enol 15 (1 equiv) were successively added and the reaction mixture was stirred 4 days at rt. ${ }^{b}$ Procedure for the synthesis of 19s and 201-r: Enol-Ugi adduct 17 (1 equiv) and iron powder (24 equiv) were stirred at $\mathrm{rt}$ in acetic acid for $2-4 \mathrm{~h} .{ }^{\mathrm{c}}$ All yields refer to isolated yields.

On the other hand, the $\beta$-alanine-derived adduct (17s) cyclizes again by attack on the amide to give pyrazine 19s. This reaction is more favorable than cyclization on the ester, as this would involve the formation of a seven-membered, instead of a six membered ring.

\section{Materials and Methods}

\subsection{Starting Materials}

Acetonitrile was dried by distillation over $\mathrm{P}_{2} \mathrm{O}_{5}$, immediately prior to use. Glacial acetic acid was purchased from commercial sources and used as received. Aldehydes $(\mathbf{1 2} \mathbf{a}-\mathbf{e})$, amines $(\mathbf{1 3 a} \mathbf{a}-\mathbf{f})$, isocyanides (14a-d; S.I. Figure S1), 4-hydroxycoumarin and iron powder are commercially available and were used without purification. 4-Hydroxy-3nitrocoumarin (15) was prepared by nitration of 4-hydroxycoumarin [52]. Imines (16a-k; S.I. Figure S2) were synthesized using the standard procedure of mixing equimolar amounts of the corresponding aldehydes (12), amines (13) and anhydrous $\mathrm{Na}_{2} \mathrm{SO}_{4}$ in $\operatorname{dry~} \mathrm{CH}_{2} \mathrm{Cl}_{2}$ at room temperature for $24 \mathrm{~h}$. [53,54] Evaporation of the solvent quantitatively yielded the imines (16) that were used in the Ugi reaction without further purification. 


\subsection{General Synthetic Techniques}

Liquid reagents were measured using positive-displacement micropipettes with disposable tips and pistons. Thin layer chromatography was performed on aluminum plates, using $254 \mathrm{~nm}$ UV light or a mixture of $p$-anisaldehyde $(2.5 \%)$, acetic acid $(1 \%)$ and $\mathrm{H}_{2} \mathrm{SO}_{4}$ $(3.4 \%)$ in $95 \%$ ethanol as developer.

\subsection{Instrumentation}

Melting points are uncorrected. IR spectra were recorded as $\mathrm{KBr}$ pellets. Proton and carbon-13 nuclear magnetic resonance $\left({ }^{1} \mathrm{H}-\mathrm{NMR}\right.$ or $\left.{ }^{13} \mathrm{C}-\mathrm{NMR}\right)$ spectra were obtained on a $500 \mathrm{MHz}$ spectrometer. The assignments of signals in ${ }^{13} \mathrm{C}$-NMR were made by DEPT. High resolution mass spectra (HRMS) were recorded using a 6520 Accurate Mass QTOF LC/MS Spectrometer.

\subsection{Synthesis and Characterization of the Ugi-Aducts \\ 3.4.1. Three-Component Condensation}

Our previously reported procedure [51] was followed. Briefly, isocyanide (14, 0.5 $\mathrm{mmol})$ and enol $(\mathbf{1 5}, 0.5 \mathrm{mmol})$ were successively added to a solution of imine (16, 0.5 $\mathrm{mmol})$ in $\mathrm{CH}_{2} \mathrm{Cl}_{2}(1 \mathrm{~mL})$, and the resulting mixture was stirred at $20^{\circ} \mathrm{C}$ for $3 \mathrm{~h}$. Removal of the solvent and purification by column chromatography $\left(\mathrm{SiO}_{2}\right.$, gradient from $100 \%$ hexanes to hexanes-EtOAc, 7:3) gave the corresponding enamines 17a-k (Table 1).

2-(Benzyl(3-nitro-2-oxo-2H-chromen-4-yl)amino)-2-(2-bromophenyl)-N-cyclohexyl acetamide (17b). Obtained from isocyanide 14a, enol 15 and imine 16b, from isocyanide 14a, enol 15 and imine $16 \mathrm{~b}$, as a yellow solid $(260 \mathrm{mg}, 88 \%)$; m.p. $165-167^{\circ} \mathrm{C}$; IR $\left(\mathrm{cm}^{-1}\right) 3341,3064$, 2933, 2854, 1714, 1677, 1600, 1549, 1465, 1350, 1278, 1116, 1054, 925, 760; ${ }^{1} \mathrm{H}-\mathrm{NMR}(500 \mathrm{MHz}$, $\left.\mathrm{CDCl}_{3}\right) \delta 8.14(\mathrm{~d}, J=8.0 \mathrm{~Hz}, 1 \mathrm{H}), 7.63-7.57(\mathrm{~m}, 3 \mathrm{H}), 7.42(\mathrm{dt}, J=7.5,0.9 \mathrm{~Hz}, 1 \mathrm{H}), 7.40(\mathrm{dt}, J$ $=3.7,1.0 \mathrm{~Hz}, 1 \mathrm{H}), 7.38-7.36(\mathrm{~m}, 1 \mathrm{H}), 7.31(\mathrm{dd}, J=8.3,0.8 \mathrm{~Hz}, 1 \mathrm{H}), 7.28(\mathrm{dd}, J=7.8,1.4 \mathrm{~Hz}$, $1 \mathrm{H}), 7.25-7.18(\mathrm{~m}, 5 \mathrm{H}), 5.87(\mathrm{~s}, 1 \mathrm{H}), 5.56(\mathrm{~d}, J=8.0 \mathrm{~Hz}, 1 \mathrm{H}), 4.77(\mathrm{~d}, J=14.8 \mathrm{~Hz}, 1 \mathrm{H}), 3.99$ $(\mathrm{d}, J=14.8 \mathrm{~Hz}, 1 \mathrm{H}), 3.82-3.73(\mathrm{~m}, 1 \mathrm{H}), 1.93-1.54(\mathrm{~m}, 5 \mathrm{H}), 1.39-0.97(\mathrm{~m}, 5 \mathrm{H}) ;{ }^{13} \mathrm{C}-\mathrm{NMR}(126$ $\mathrm{MHz}, \mathrm{CDCl}_{3}$ ) $\delta 167.45$ (C), 155.12 (C), 153.00 (C), 152.48 (C), 135.14 (C), 134.33 (C), 133.96 $(\mathrm{CH}), 133.82(\mathrm{CH}), 130.99(\mathrm{CH}), 130.13(\mathrm{CH}), 129.02(\mathrm{CH}), 128.61(\mathrm{CH}), 128.44(\mathrm{CH}), 128.24$ $(\mathrm{CH}), 127.49(\mathrm{CH}), 126.33(\mathrm{C}), 125.12(\mathrm{CH}), 118.45(\mathrm{C}), 117.90(\mathrm{CH}), 68.92(\mathrm{CH}), 54.76\left(\mathrm{CH}_{2}\right)$, $49.26(\mathrm{CH}), 32.73\left(\mathrm{CH}_{2}\right), 32.71\left(\mathrm{CH}_{2}\right), 24.82\left(\mathrm{CH}_{2}\right), 24.74\left(\mathrm{CH}_{2}\right) ; \mathrm{MS}(\mathrm{qTOF}) \mathrm{m} / z(\%) 592$ $\left(\mathrm{M}^{+}+3,28\right), 590\left(\mathrm{M}^{+}+1,30\right), 574(42), 572$ (44), 510 (98), 508 (100); HRMS (qTOF) Calcd for $\mathrm{C}_{30} \mathrm{H}_{29} \mathrm{BrN}_{3} \mathrm{O}_{5}$ : 590.1296. Found: 590.1285.

2-(Benzyl(3-nitro-2-oxo-2H-chromen-4-yl)amino)-N-cyclohexyl-2-(p-tolyl)acetamide (17c). Obtained from isocyanide 14a, enol 15 and imine 16c, as a yellow solid (208 mg, 79\%); m.p. $141-143{ }^{\circ} \mathrm{C}$; IR $\left(\mathrm{cm}^{-1}\right) 3369,2928,2853,1729,1681,1601,1549,1451,1403,1350,1116,1054$, 791; ${ }^{1} \mathrm{H}-\mathrm{NMR}\left(500 \mathrm{MHz}, \mathrm{CDCl}_{3}\right) \delta 8.04(\mathrm{~d}, J=8.1 \mathrm{~Hz}, 1 \mathrm{H}), 7.56(\mathrm{dt}, J=7.3,1.4 \mathrm{~Hz}, 1 \mathrm{H})$, $7.31-7.26(\mathrm{~m}, 2 \mathrm{H}), 7.24-7.11(\mathrm{~m}, 9 \mathrm{H}), 5.78(\mathrm{~d}, J=8.1 \mathrm{~Hz}, 1 \mathrm{H}), 5.16(\mathrm{~s}, 1 \mathrm{H}), 4.64(\mathrm{~d}, J=14.9 \mathrm{~Hz}$, $1 \mathrm{H}), 4.19(\mathrm{~d}, J=14.9 \mathrm{~Hz}, 1 \mathrm{H}), 3.76-3.67(\mathrm{~m}, 1 \mathrm{H}), 2.33(\mathrm{~s}, 3 \mathrm{H}), 1.87-1.53(\mathrm{~m}, 5 \mathrm{H}), 1.36-0.95$ $(\mathrm{m}, 5 \mathrm{H}) ;{ }^{13} \mathrm{C}-\mathrm{NMR}\left(126 \mathrm{MHz}, \mathrm{CDCl}_{3}\right) \delta 168.30$ (C), 155.08 (C), 153.26 (C), 152.49 (C), 139.50 (C), $135.31(\mathrm{C}), 133.89(\mathrm{CH}), 131.78(\mathrm{C}), 129.90(\mathrm{CH}), 129.16(\mathrm{CH}), 129.04(\mathrm{CH}), 128.57(\mathrm{C})$, $128.45(\mathrm{CH}), 128.39(\mathrm{CH}), 124.93(\mathrm{CH}), 118.38(\mathrm{C}), 117.65(\mathrm{CH}), 71.15(\mathrm{CH}), 55.96\left(\mathrm{CH}_{2}\right)$, $48.99(\mathrm{CH}), 32.79\left(\mathrm{CH}_{2}\right), 32.70\left(\mathrm{CH}_{2}\right), 27.07\left(\mathrm{CH}_{2}\right), 24.87\left(\mathrm{CH}_{2}\right), 24.79\left(\mathrm{CH}_{2}\right), 21.32\left(\mathrm{CH}_{3}\right)$; MS (qTOF) $m / z(\%) 526\left(\mathrm{M}^{+}+1,<5\right), 479$ (10), 347 (100), 146 (54); HRMS (qTOF) Calcd for $\mathrm{C}_{33} \mathrm{H}_{32} \mathrm{~N}_{3} \mathrm{O}_{5}$ : 526.2326. Found: 526.2326.

2-(Benzyl(3-nitro-2-oxo-2H-chromen-4-yl)amino)-N-cyclohexyl-2-(4-(trifluoromethyl) phenyl) acetamide (17d). Obtained from isocyanide 14a, enol 15 and imine $\mathbf{1 6 d}$, as a yellow solid (226 mg, 78\%), m.p. $135-137^{\circ} \mathrm{C}$; IR $\left(\mathrm{cm}^{-1}\right) 3365,2932,2855,1730,1684,1603,1550,1324$, $1168,1127,1068,761,699 ;{ }^{1} \mathrm{H}-\mathrm{NMR}\left(500 \mathrm{MHz}, \mathrm{CDCl}_{3}\right) \delta 7.90(\mathrm{~d}, J=7.3 \mathrm{~Hz}, 1 \mathrm{H}), 7.61-7.57$ $(\mathrm{m}, 3 \mathrm{H}), 7.52(\mathrm{~d}, J=8.2 \mathrm{~Hz}, 2 \mathrm{H}), 7.30(\mathrm{~d}, J=7.7 \mathrm{~Hz}, 2 \mathrm{H}), 7.24-7.18(\mathrm{~m}, 3 \mathrm{H}), 7.10(\mathrm{dd}, J=6.5$, $1.6 \mathrm{~Hz}, 2 \mathrm{H}), 5.88(\mathrm{~d}, J=8.1 \mathrm{~Hz}, 1 \mathrm{H}), 5.16(\mathrm{~s}, 1 \mathrm{H}), 4.63(\mathrm{~d}, J=14.7 \mathrm{~Hz}, 1 \mathrm{H}), 4.23(\mathrm{~d}, J=14.7 \mathrm{~Hz}$, $1 \mathrm{H}), 3.71-3.61(\mathrm{~m}, 1 \mathrm{H}), 1.84-1.55(\mathrm{~m}, 5 \mathrm{H}), 1.35-0.93(\mathrm{~m}, 5 \mathrm{H}) ;{ }^{13} \mathrm{C}-\mathrm{NMR}\left(126 \mathrm{MHz}, \mathrm{CDCl}_{3}\right) \delta$ 167.30 (C), 154.79 (C), 153.12 (C), 152.48 (C), 139.01 (C), 134.71 (C), $134.23(\mathrm{CH}), 131.76$ (C), 
$131.50(\mathrm{C}), 129.35(\mathrm{CH}), 129.29(\mathrm{CH}), 128.75(\mathrm{CH}), 128.70(\mathrm{CH}), 128.28(\mathrm{CH}), 126.15(\mathrm{CH})$, $126.12(\mathrm{CH}), 125.05(\mathrm{CH}), 122.73(\mathrm{C}), 118.21(\mathrm{C}), 117.80(\mathrm{CH}), 70.56(\mathrm{CH}), 56.77\left(\mathrm{CH}_{2}\right), 49.15$ $(\mathrm{CH}), 32.68\left(\mathrm{CH}_{2}\right), 32.60\left(\mathrm{CH}_{2}\right), 25.41\left(\mathrm{CH}_{2}\right), 24.82\left(\mathrm{CH}_{2}\right), 24.75\left(\mathrm{CH}_{2}\right) ; \mathrm{MS}(\mathrm{qTOF}) \mathrm{m} / z(\%)$ $580\left(\mathrm{M}^{+}+1,100\right), 391$ (13), 309 (28); HRMS (qTOF) Calcd for $\mathrm{C}_{31} \mathrm{H}_{29} \mathrm{~F}_{3} \mathrm{~N}_{3} \mathrm{O}_{5}: 580.2059$. Found: 580.2059 .

2-((Benzo[d][1,3]dioxol-5-ylmethyl)(3-nitro-2-oxo-2H-chromen-4-yl)amino)-N-cyclohexyl-2phenylacetamide (17e). Obtained from isocyanide 14a, enol 15 and imine 16e, as a yellow solid (217 mg, 78\%); m.p. 135-136 ${ }^{\circ} \mathrm{C}$; IR ( $\left.\mathrm{cm}^{-1}\right)$ 3369, 2930, 2853, 1728, 1681, 1601, 1549, 1504, 1489, 1446, 1401, 1347, 1249, 929, 761; ${ }^{1} \mathrm{H}-\mathrm{NMR}\left(500 \mathrm{MHz}, \mathrm{CDCl}_{3}\right) \delta 8.07(\mathrm{dd}, J=8.1$, $1.3 \mathrm{~Hz}, 1 \mathrm{H}), 7.56(\mathrm{dt}, J=6.9,1.4 \mathrm{~Hz}, 1 \mathrm{H}), 7.38-7.28(\mathrm{~m}, 7 \mathrm{H}), 6.74(\mathrm{~d}, J=1.4 \mathrm{~Hz}, 1 \mathrm{H}), 6.61(\mathrm{~d}, J$ $=7.9, \mathrm{~Hz}, 1 \mathrm{H}), 6.57(\mathrm{dd}, J=8.0,1.5 \mathrm{~Hz}, 1 \mathrm{H}), 5.89(\mathrm{~s}, 2 \mathrm{H}), 5.78(\mathrm{~d}, J=8.1 \mathrm{~Hz}, 1 \mathrm{H}), 5.19(\mathrm{~s}$, $1 \mathrm{H}), 4.56(\mathrm{~d}, J=14.8 \mathrm{~Hz}, 1 \mathrm{H}), 4.09(\mathrm{~d}, J=14.8 \mathrm{~Hz}, 1 \mathrm{H}), 3.76-3.67(\mathrm{~m}, 1 \mathrm{H}), 1.89-1.53(\mathrm{~m}, 5 \mathrm{H})$, 1.37-0.95 (m, 5H); ${ }^{13} \mathrm{C}-\mathrm{NMR}\left(126 \mathrm{MHz} \mathrm{CDCl}_{3}\right) \delta 168.11(\mathrm{C}), 155.06(\mathrm{C}), 152.50(\mathrm{C}), 147.83$ (C), $147.73(\mathrm{C}), 134.78(\mathrm{C}), 134.00(\mathrm{CH}), 129.52(\mathrm{CH}), 129.23(\mathrm{CH}), 129.12(\mathrm{CH}), 128.91(\mathrm{C})$, $128.31(\mathrm{CH}), 125.06(\mathrm{CH}), 122.90(\mathrm{CH}), 118.25(\mathrm{C}), 117.71(\mathrm{CH}), 109.38(\mathrm{CH}), 108.16(\mathrm{CH})$, $101.22\left(\mathrm{CH}_{2}\right), 71.29(\mathrm{CH}), 55.71\left(\mathrm{CH}_{2}\right), 49.04(\mathrm{CH}), 32.76\left(\mathrm{CH}_{2}\right), 32.67\left(\mathrm{CH}_{2}\right), 27.05\left(\mathrm{CH}_{2}\right)$, $24.85\left(\mathrm{CH}_{2}\right), 24.77\left(\mathrm{CH}_{2}\right)$; MS (qTOF) $m / z(\%) 556\left(\mathrm{M}^{+}+1,672\right), 457(20), 353(100)$; HRMS (qTOF) Calcd for $\mathrm{C}_{31} \mathrm{H}_{30} \mathrm{~N}_{3} \mathrm{O}_{7}: 556.2084$. Found: 556.2089.

2-((Benzo[d][1,3]dioxol-5-ylmethyl)(3-nitro-2-oxo-2H-chromen-4-yl)amino)-N-cyclohexyl-2( $p$-tolyl)acetamide (17f). Obtained from isocyanide 14a, enol 15 and imine 16f, as a yellow solid (202 mg, 71\%); m.p. 145-146 ${ }^{\circ} \mathrm{C}$; IR ( $\left.\mathrm{cm}^{-1}\right)$ 3424, 2929, 2853, 1728, 1679, 1601, 1549, 1489, 1446, 1249, 1039, 929, 761; ${ }^{1} \mathrm{H}-\mathrm{NMR}\left(500 \mathrm{MHz}, \mathrm{CDCl}_{3}\right) \delta 8.09$ (d, J = 7.2 Hz, 1H), 7.58 $(\mathrm{dt}, J=6.4,1.3 \mathrm{~Hz}, 1 \mathrm{H}), 7.35-7.27(\mathrm{~m}, 2 \mathrm{H}), 7.21(\mathrm{~d}, J=8.0 \mathrm{~Hz}, 2 \mathrm{H}), 7.14(\mathrm{~d}, J=7.9 \mathrm{~Hz}, 2 \mathrm{H})$, $6.75(\mathrm{~s}, 1 \mathrm{H}), 6.63-6.55(\mathrm{~m}, 2 \mathrm{H}), 5.88(\mathrm{~s}, 2 \mathrm{H}), 5.72(\mathrm{~d}, J=8.1 \mathrm{~Hz}, 1 \mathrm{H}), 5.16(\mathrm{~s}, 1 \mathrm{H}), 4.55(\mathrm{~d}, J$ $=14.8 \mathrm{~Hz}, 1 \mathrm{H}), 4.08(\mathrm{~d}, J=15.0 \mathrm{~Hz}, 1 \mathrm{H}), 3.77-3.68(\mathrm{~m}, 1 \mathrm{H}), 2.33(\mathrm{~s}, 3 \mathrm{H}), 1.88-1.54(\mathrm{~m}, 5 \mathrm{H})$, 1.35-0.95 (m, 5H); ${ }^{13} \mathrm{C}-\mathrm{NMR}\left(126 \mathrm{MHz} \mathrm{CDCl}_{3}\right) \delta 168.36(\mathrm{C}), 153.15(\mathrm{C}), 152.55(\mathrm{C}), 147.84$ (C), $147.71(\mathrm{C}), 139.54(\mathrm{C}), 133.92(\mathrm{CH}), 131.77(\mathrm{C}), 130.54(\mathrm{C}), 129.93(\mathrm{CH}), 129.07(\mathrm{CH})$, $128.36(\mathrm{CH}), 125.02(\mathrm{CH}), 122.90(\mathrm{CH}), 118.36(\mathrm{C}), 118.32(\mathrm{C}), 117.69(\mathrm{CH}), 109.43(\mathrm{CH})$, $108.15(\mathrm{CH}), 101.20\left(\mathrm{CH}_{2}\right), 71.14(\mathrm{CH}), 55.58\left(\mathrm{CH}_{2}\right), 49.04(\mathrm{CH}), 32.82\left(\mathrm{CH}_{2}\right), 32.73\left(\mathrm{CH}_{2}\right)$, $27.07\left(\mathrm{CH}_{2}\right), 24.88\left(\mathrm{CH}_{2}\right), 24.80\left(\mathrm{CH}_{2}\right), 21.32\left(\mathrm{CH}_{3}\right)$; MS (qTOF) $m / z(\%) 570\left(\mathrm{M}^{+}+1,70\right)$, 353 (100); HRMS (qTOF) Calcd for $\mathrm{C}_{32} \mathrm{H}_{32} \mathrm{~N}_{3} \mathrm{O}_{7}$ : 570.2240. Found: 570.2236.

2-((Benzo[d][1,3]dioxol-5-ylmethyl)(3-nitro-2-oxo-2H-chromen-4-yl)amino)-N-cyclohexyl-2(4-(trifluoromethyl)phenyl)acetamide $(\mathbf{1 7 g})$. Obtained from isocyanide $\mathbf{1 4 a}$, enol $\mathbf{1 5}$ and imine 16g, as a yellow solid (250 mg, 80\%); m.p. 129-131 ${ }^{\circ} \mathrm{C}$; IR $\left(\mathrm{cm}^{-1}\right) 3362,2931,2854,1727$, 1683, 1603, 1550, 1490, 1447, 1324, 1250, 1167, 1127, 1068, 930, 761; ${ }^{1} \mathrm{H}-\mathrm{NMR}(500 \mathrm{MHz}$, $\left.\mathrm{CDCl}_{3}\right) \delta 7.96(\mathrm{dd}, J=8.1,1.1 \mathrm{~Hz}, 1 \mathrm{H}), 7.64-7.56(\mathrm{~m}, 3 \mathrm{H}), 7.52(\mathrm{~d}, J=8.2 \mathrm{~Hz}, 2 \mathrm{H}), 7.35-7.29$ $(\mathrm{m}, 2 \mathrm{H}), 6.67(\mathrm{~d}, J=1.5 \mathrm{~Hz}, 1 \mathrm{H}), 6.62(\mathrm{~d}, J=7.9 \mathrm{~Hz}, 1 \mathrm{H}), 6.52(\mathrm{dd}, J=8.0,1.5 \mathrm{~Hz}, 1 \mathrm{H}), 5.90$ $(\mathrm{s}, 2 \mathrm{H}), 5.82(\mathrm{~d}, J=8.0 \mathrm{~Hz}, 1 \mathrm{H}), 5.16(\mathrm{~s}, 1 \mathrm{H}), 4.55(\mathrm{~d}, J=14.6 \mathrm{~Hz}, 1 \mathrm{H}), 4.16(\mathrm{~d}, J=14.6 \mathrm{~Hz}$, $1 \mathrm{H}), 3.72-3.63(\mathrm{~m}, 1 \mathrm{H}), 1.87-1.52(\mathrm{~m}, 5 \mathrm{H}), 1.35-0.94(\mathrm{~m}, 5 \mathrm{H}) ;{ }^{13} \mathrm{C}-\mathrm{NMR}\left(126 \mathrm{MHz}, \mathrm{CDCl}_{3}\right) \delta$ $167.34(\mathrm{C}), 154.78(\mathrm{C}), 153.00(\mathrm{C}), 152.53(\mathrm{C}), 148.00$ (C), 147.96 (C), 138.97 (C), 134.28 (CH), $129.35(\mathrm{CH}), 128.39(\mathrm{C}), 128.20(\mathrm{CH}), 126.17(\mathrm{CH}), 126.14(\mathrm{CH}), 125.14(\mathrm{CH}), 123.07(\mathrm{CH})$, $118.16(\mathrm{C}), 117.85(\mathrm{CH}), 109.48(\mathrm{CH}), 108.28(\mathrm{CH}), 101.34\left(\mathrm{CH}_{2}\right), 70.52(\mathrm{CH}), 56.42\left(\mathrm{CH}_{2}\right)$, $49.19(\mathrm{CH}), 32.71\left(\mathrm{CH}_{2}\right), 32.64\left(\mathrm{CH}_{2}\right), 27.07\left(\mathrm{CH}_{2}\right), 24.83\left(\mathrm{CH}_{2}\right), 24.76\left(\mathrm{CH}_{2}\right) ; \mathrm{MS}(\mathrm{qTOF}) \mathrm{m} / z$ (\%) $624\left(\mathrm{M}^{+}+1,35\right), 353$ (100); HRMS (qTOF) Calcd for $\mathrm{C}_{32} \mathrm{H}_{29} \mathrm{~F}_{3} \mathrm{~N}_{3} \mathrm{O}_{7}$ : 624.1958. Found: 624.1942.

N-Cyclohexyl-2-(cyclohexyl(3-nitro-2-oxo-2H-chromen-4-yl)amino)-2-(p-tolyl)acetamide (17h). Obtained from isocyanide 14a, enol 15 and imine 16h, as a pale orange solid (205 mg, 79\%); m.p. $68-70{ }^{\circ} \mathrm{C}$; IR $\left(\mathrm{cm}^{-1}\right)$ 3415, 2931, 2855, 1736, 1662, 1605, 1540, 1451, 1374, 1276, 1111, 1055, 762; ${ }^{1} \mathrm{H}-\mathrm{NMR}\left(500 \mathrm{MHz}, \mathrm{CDCl}_{3}\right) \delta 7.98$ (bs, $\left.1 \mathrm{H}\right), 7.53(\mathrm{dt}, J=7.2,1.3 \mathrm{~Hz}, 1 \mathrm{H}), 7.32-7.28(\mathrm{~m}$, $3 \mathrm{H}), 7.21(\mathrm{~d}, J=7.6 \mathrm{~Hz}, 1 \mathrm{H}), 6.90(\mathrm{~d}, J=8.0 \mathrm{~Hz}, 2 \mathrm{H}), 6.53(\mathrm{~d}, J=5.8 \mathrm{~Hz}, 1 \mathrm{H}), 5.07(\mathrm{~s}, 1 \mathrm{H})$, 3.72-3.61 (m, 1H), $3.32(\mathrm{tt}, J=11.6,3.4 \mathrm{~Hz}, 1 \mathrm{H}), 2.17(\mathrm{~s}, 3 \mathrm{H}), 2.10-0.81(\mathrm{~m}, 20 \mathrm{H}) ;{ }^{13} \mathrm{C}-\mathrm{NMR}(126$ $\mathrm{MHz}_{\mathrm{CDCl}}$ ) $\delta 170.63(\mathrm{C}), 154.63(\mathrm{C}), 153.53(\mathrm{C}), 151.63(\mathrm{C}), 138.79(\mathrm{C}), 133.82(\mathrm{CH}), 132.54$ (C), $129.27(\mathrm{CH}), 129.21(\mathrm{CH}), 129.12(\mathrm{CH}), 128.70(\mathrm{C}), 127.78(\mathrm{CH}), 127.12(\mathrm{CH}), 124.57(\mathrm{CH})$, $120.12(\mathrm{C}), 117.24(\mathrm{CH}), 72.17(\mathrm{CH}), 66.58(\mathrm{CH}), 48.60(\mathrm{CH}), 32.73\left(\mathrm{CH}_{2}\right), 32.63\left(\mathrm{CH}_{2}\right), 32.45$ 
$\left(\mathrm{CH}_{2}\right), 32.28\left(\mathrm{CH}_{2}\right), 27.06\left(\mathrm{CH}_{2}\right), 26.09\left(\mathrm{CH}_{2}\right), 26.08\left(\mathrm{CH}_{2}\right), 24.97\left(\mathrm{CH}_{2}\right), 24.94\left(\mathrm{CH}_{2}\right), 21.14$ $\left(\mathrm{CH}_{3}\right)$; MS (qTOF) $m / z$ (\%) $518\left(\mathrm{M}^{+}+1,30\right), 517$ (35), 447 (100); HRMS (qTOF) Calcd for $\mathrm{C}_{30} \mathrm{H}_{36} \mathrm{~N}_{3} \mathrm{O}_{5}$ : 518.2655. Found: 518.2648.

$\mathrm{N}$-Cyclohexyl-2-(cyclohexyl(3-nitro-2-oxo-2H-chromen-4-yl)amino)-2-(4-(trifluoromethyl) phenyl)acetamide (17i). Obtained from isocyanide 14a, enol 15 and imine 16i, as a pale orange solid (243 mg, 85\%); m.p. 133-135 ${ }^{\circ} \mathrm{C}$; IR $\left(\mathrm{cm}^{-1}\right) 3386,2933,2856,1737,1681,1606$, $1541,1325,1167,1127,1068,762 ;{ }^{1} \mathrm{H}-\mathrm{NMR}\left(500 \mathrm{MHz}, \mathrm{CDCl}_{3}\right) \delta 7.93$ (bs, $\left.1 \mathrm{H}\right), 7.59$ (d, J = 8.2 $\mathrm{Hz}, 2 \mathrm{H}), 7.54(\mathrm{t}, J=7.3 \mathrm{~Hz}, 1 \mathrm{H}), 7.40(\mathrm{~d}, J=8.2 \mathrm{~Hz}, 2 \mathrm{H}), 7.32(\mathrm{t}, J=7.7 \mathrm{~Hz}, 1 \mathrm{H}), 7.24(\mathrm{~d}, J$ $=8.3 \mathrm{~Hz}, 1 \mathrm{H}), 6.55(\mathrm{bs}, 1 \mathrm{H}), 5.16(\mathrm{~s}, 1 \mathrm{H}), 3.68-3.57(\mathrm{~m}, 1 \mathrm{H}), 3.30(\mathrm{tt}, J=11.6,3.4 \mathrm{~Hz}, 1 \mathrm{H})$, 2.09-1.53 (m, 10H), 1.37-0.95 (m, 10H); ${ }^{13} \mathrm{C}-\mathrm{NMR}\left(126 \mathrm{MHz}, \mathrm{CDCl}_{3}\right) \delta 169.44(\mathrm{C}), 154.32(\mathrm{C})$, $152.78(\mathrm{C}), 151.79(\mathrm{C}), 140.01(\mathrm{C}), 134.25(\mathrm{CH}), 128.81(\mathrm{CH}), 125.58(\mathrm{CH}), 125.55(\mathrm{CH}), 124.86$ $(\mathrm{CH}), 119.84(\mathrm{C}), 117.56(\mathrm{CH}), 72.08(\mathrm{CH}), 66.63(\mathrm{CH}), 48.84(\mathrm{CH}), 32.66\left(\mathrm{CH}_{2}\right), 32.58\left(\mathrm{CH}_{2}\right)$, $32.49\left(\mathrm{CH}_{2}\right), 32.30\left(\mathrm{CH}_{2}\right), 27.07\left(\mathrm{CH}_{2}\right), 26.07\left(\mathrm{CH}_{2}\right), 25.47\left(\mathrm{CH}_{2}\right), 25.23\left(\mathrm{CH}_{2}\right), 24.92\left(\mathrm{CH}_{2}\right)$, $24.89\left(\mathrm{CH}_{2}\right) ; \mathrm{MS}$ (qTOF) $m / z(\%) 572\left(\mathrm{M}^{+}+1,69\right), 473$ (100), 383 (17), 301 (171); HRMS (qTOF) Calcd for $\mathrm{C}_{30} \mathrm{H}_{33} \mathrm{~F}_{3} \mathrm{~N}_{3} \mathrm{O}_{5}: 572.2372$. Found: 572.2367 .

$\mathrm{N}$-Cyclohexyl-2-(3,4-dimethoxyphenyl)-2-((3-nitro-2-oxo-2H-chromen-4-yl)(phenyl)amino) acetamide (17k). Obtained from isocyanide 14a, enol 15 and imine 16k, as an orange solid (112 $\mathrm{mg}, 40 \%)$; m.p. $129-130^{\circ} \mathrm{C}$; IR $\left(\mathrm{cm}^{-1}\right) 3403,2931,2853,1740,1681,1604,1517,1451,1373$, 1265, 1148, 761; ${ }^{1} \mathrm{H}-\mathrm{NMR}\left(500 \mathrm{MHz}, \mathrm{CDCl}_{3}\right) \delta 7.67(\mathrm{~d}, J=7.0 \mathrm{~Hz}, 1 \mathrm{H}), 7.55(\mathrm{dt}, J=6.1,1.5$ $\mathrm{Hz}, 1 \mathrm{H}), 7.29-7.20(\mathrm{~m}, 5 \mathrm{H}), 7.03(\mathrm{t}, J=7.4 \mathrm{~Hz}, 1 \mathrm{H}), 6.99(\mathrm{~d}, J=8.0 \mathrm{~Hz}, 2 \mathrm{H}), 6.76(\mathrm{dd}, J=8.3$, $2.1 \mathrm{~Hz}, 2 \mathrm{H}), 6.67(\mathrm{~d}, J=1.9 \mathrm{~Hz}, 2 \mathrm{H}), 6.61(\mathrm{~d}, J=8.4 \mathrm{~Hz}, 2 \mathrm{H}), 6.37(\mathrm{~d}, J=8.2 \mathrm{~Hz}, 2 \mathrm{H}), 5.50$ $(\mathrm{s}, 2 \mathrm{H}), 3.87-3.78(\mathrm{~m}, 2 \mathrm{H}), 3.77(\mathrm{~s}, 3 \mathrm{H}), 3.58(\mathrm{~s}, 3 \mathrm{H}), 1.94-1.52(\mathrm{~m}, 5 \mathrm{H}), 1.39-0.94(\mathrm{~m}, 5 \mathrm{H})$; ${ }^{13} \mathrm{C}-\mathrm{NMR}\left(126 \mathrm{MHz}, \mathrm{CDCl}_{3}\right) \delta 168.15$ (C), 153.89 (C), 152.90 (C), 150.26 (C), 149.83 (C), 148.68 (C), $144.87(\mathrm{C}), 134.24(\mathrm{CH}), 129.75(\mathrm{CH}), 128.78(\mathrm{CH}), 125.29(\mathrm{CH}), 124.44(\mathrm{C}), 123.13(\mathrm{CH})$, $123.04(\mathrm{CH}), 118.23(\mathrm{C}), 117.81(\mathrm{CH}), 117.60(\mathrm{CH}), 112.72(\mathrm{CH}), 110.84(\mathrm{CH}), 69.97(\mathrm{CH}), 55.90$ $\left(\mathrm{CH}_{3}\right), 55.65\left(\mathrm{CH}_{3}\right), 48.64(\mathrm{CH}), 32.83\left(\mathrm{CH}_{2}\right), 32.43\left(\mathrm{CH}_{2}\right), 25.43\left(\mathrm{CH}_{2}\right), 24.71\left(\mathrm{CH}_{2}\right), 24.58$ $\left(\mathrm{CH}_{2}\right)$; MS (qTOF) $m / z(\%) 558\left(\mathrm{M}^{+}+1,<5\right), 478$ (35), 328 (57); HRMS (qTOF) Calcd for $\mathrm{C}_{31} \mathrm{H}_{32} \mathrm{~N}_{3} \mathrm{O}_{7}$ : 558.2240. Found: 558.2234.

\subsubsection{Four-Component Condensation}

Amine $13(0.5 \mathrm{mmol})$ was added to a solution of aldehyde $12(0.5 \mathrm{mmol})$ in of dry acetonitrile $(1 \mathrm{~mL})$. The resulting mixture was stirred for $15 \mathrm{~min}$ at $\mathrm{rt}$ and then isocyanide $14(0.5 \mathrm{mmol})$ and enol $15(0.5 \mathrm{mmol})$ were successively added. After 4 days stirring at room temperature, the reaction went to completion, as judged by tlc. Then $10 \% \mathrm{HCl}$ (2 $\mathrm{mL}$ ) was added, the mixture was washed with $\mathrm{H}_{2} \mathrm{O}(15 \mathrm{~mL})$, extracted with $\mathrm{CH}_{2} \mathrm{Cl}_{2}$ (3 $\times 20 \mathrm{~mL}$ ) and dried over $\mathrm{Na}_{2} \mathrm{SO}_{4}$. Removal of the solvent and purification by column chromatography $\left(\mathrm{SiO}_{2}\right.$, gradient from $100 \%$ hexanes to hexanes-EtOAc, 7:3) gave the corresponding enamines 171-s (Table 2).

Methyl N-(2-(cyclohexylamino)-2-oxo-1-phenylethyl)-N-(3-nitro-2-oxo-2H-chromen-4-yl)glycinate (171). Obtained from aldehyde 12a, amine 13e, isocyanide 14a and enol 15, as a pale orange solid (141 mg, 57\%); m.p. 132-134 ${ }^{\circ} \mathrm{C}$; IR ( $\left.\mathrm{cm}^{-1}\right)$ 3359, 2931, 2854, 1730, 1681, 1606, 1554, 1452, 1212,$760 ;{ }^{1} \mathrm{H}-\mathrm{NMR}\left(500 \mathrm{MHz}, \mathrm{CDCl}_{3}\right) \delta 8.12(\mathrm{~d}, J=8.1 \mathrm{~Hz}, 1 \mathrm{H}), 7.63(\mathrm{t}, J=7.7 \mathrm{~Hz}, 1 \mathrm{H}), 7.44-7.33$ $(\mathrm{m}, 5 \mathrm{H}), 5.90(\mathrm{~d}, J=8.0 \mathrm{~Hz}, 1 \mathrm{H}), 5.39(\mathrm{~s}, 1 \mathrm{H}), 4.10(\mathrm{~d}, J=18.0 \mathrm{~Hz}, 1 \mathrm{H}), 3.80(\mathrm{~d}, J=18.0 \mathrm{~Hz}, 1 \mathrm{H})$, 3.74-3.66 (m, 1H), $3.64(\mathrm{~s}, 3 \mathrm{H}), 1.91-0.93(\mathrm{~m}, 10 \mathrm{H}) ;{ }^{13} \mathrm{C}-\mathrm{NMR}\left(126 \mathrm{MHz}, \mathrm{CDCl}_{3}\right) \delta 169.12(\mathrm{C})$, $167.52(\mathrm{C}), 152.60(\mathrm{C}), 152.42(\mathrm{C}), 134.39(\mathrm{C}), 133.94(\mathrm{CH}), 129.57(\mathrm{CH}), 129.24(\mathrm{CH}), 128.91(\mathrm{CH})$, $127.89(\mathrm{CH}), 125.15(\mathrm{CH}), 117.77(\mathrm{CH}), 70.24(\mathrm{CH}), 52.37\left(\mathrm{CH}_{3}\right), 51.80\left(\mathrm{CH}_{2}\right), 48.85(\mathrm{CH}), 32.60$ $\left(\mathrm{CH}_{2}\right), 32.53\left(\mathrm{CH}_{2}\right), 26.94\left(\mathrm{CH}_{2}\right), 25.33\left(\mathrm{CH}_{2}\right), 24.73\left(\mathrm{CH}_{2}\right), 24.66\left(\mathrm{CH}_{2}\right) ; \mathrm{MS}(\mathrm{qTOF}) \mathrm{m} / z(\%)$ $494\left(\mathrm{M}^{+}+1,26\right)$, 423 (10), 305 (100); HRMS (qTOF) Calcd for $\mathrm{C}_{26} \mathrm{H}_{28} \mathrm{~N}_{3} \mathrm{O}_{7}$ : 494.1927. Found: 494.1915.

Methyl N-(2-(cyclohexylamino)-2-oxo-1-(p-tolyl)ethyl)-N-(3-nitro-2-oxo-2H-chromen-4-yl)glycinate (17m). Obtained from aldehyde $12 \mathrm{c}$, amine $13 \mathrm{e}$, isocyanide $14 \mathrm{a}$ and enol 15 , as a pale yellow solid (122 mg, 48\%); m.p. 114-116 ${ }^{\circ} \mathrm{C}$; IR ( $\left.\mathrm{cm}^{-1}\right) 3383,2930,2854,1728,1680,1604,1551,1451$, 1209, 1119, 1057, 759; ${ }^{1} \mathrm{H}-\mathrm{NMR}\left(500 \mathrm{MHz}, \mathrm{CDCl}_{3}\right) \delta 8.09(\mathrm{dd}, J=6,99,1.5 \mathrm{~Hz}, 1 \mathrm{H}), 7.64(\mathrm{dt}, J=7.0$, $1.4 \mathrm{~Hz}, 1 \mathrm{H}), 7.40-7.33(\mathrm{~m}, 3 \mathrm{H}), 7.26-7.17(\mathrm{~m}, 3 \mathrm{H}), 5.81(\mathrm{~d}, J=8.1 \mathrm{~Hz}, 1 \mathrm{H}), 5.70(\mathrm{~s}, 1 \mathrm{H}), 4.23(\mathrm{~d}, J=$ 
$18.3 \mathrm{~Hz}, 1 \mathrm{H}), 3.82-3.71(\mathrm{~m}, 1 \mathrm{H}), 3,74(\mathrm{~d}, J=18.3 \mathrm{~Hz}, 1 \mathrm{H}), 3.66(\mathrm{~s}, 3 \mathrm{H}), 2.32(\mathrm{~s}, 3 \mathrm{H}), 1.95-1.54(\mathrm{~m}$, 5H), 1.38-0.95 (m, 5H); ${ }^{13} \mathrm{C}-\mathrm{NMR}\left(126 \mathrm{MHz} \mathrm{CDCl}_{3}\right) \delta 169.15$ (C), 168.14 (C), 155.19 (C), 152.67 (C), $152.61(\mathrm{C}), 137.90(\mathrm{C}), 134.06(\mathrm{CH}), 132.85(\mathrm{C}), 131.83(\mathrm{CH}), 129.58(\mathrm{CH}), 128.33(\mathrm{CH}), 127.79$ $(\mathrm{CH}), 126.85(\mathrm{CH}), 125.21(\mathrm{CH}), 118.07(\mathrm{CH}), 117.30(\mathrm{C}), 66.50(\mathrm{CH}), 52.62\left(\mathrm{CH}_{3}\right), 51.42\left(\mathrm{CH}_{2}\right)$, $49.00(\mathrm{CH}), 32.78\left(\mathrm{CH}_{2}\right), 32.69\left(\mathrm{CH}_{2}\right), 25.43\left(\mathrm{CH}_{2}\right), 24.87\left(\mathrm{CH}_{2}\right), 24.81\left(\mathrm{CH}_{2}\right), 19.81\left(\mathrm{CH}_{3}\right) ; \mathrm{MS}$ (qTOF) $m / z$ (\%) $508\left(\mathrm{M}^{+}+1,67\right), 319$ (100); HRMS (qTOF) Calcd for $\mathrm{C}_{27} \mathrm{H}_{30} \mathrm{~N}_{3} \mathrm{O}_{7}: 508.2084$ Found: 508.2071.

Methyl N-(2-(cyclohexylamino)-2-oxo-1-(4-(trifluoromethyl)phenyl)ethyl)-N-(3-nitro-2-oxo2 H-chromen-4-yl)glycinate (17n). Obtained from aldehyde $\mathbf{1 2 d}$, amine $\mathbf{1 3 e}$, isocyanide $\mathbf{1 4 a}$ and enol 15, as a pale yellow solid (146 mg, 52\%); m.p. $113-115^{\circ} \mathrm{C}$; IR (cm $\left.{ }^{-1}\right) 3368,2933$, 2855, 1734, 1605, 1554, 1325, 1169, 1127, 1069, 760; ${ }^{1} \mathrm{H}-\mathrm{NMR}\left(500 \mathrm{MHz}, \mathrm{CDCl}_{3}\right) \delta 8.09(\mathrm{dd}, J$ $=8.1,1.3 \mathrm{~Hz}, 1 \mathrm{H}), 7.68-7.62(\mathrm{~m}, 3 \mathrm{H}), 7.57(\mathrm{~d}, J=8.2 \mathrm{~Hz}, 2 \mathrm{H}), 7.43-7.37(\mathrm{~m}, 2 \mathrm{H}), 5.98(\mathrm{~d}, J=$ $8.1 \mathrm{~Hz}, 1 \mathrm{H}), 5.42(\mathrm{~s}, 1 \mathrm{H}), 4.14(\mathrm{~d}, J=17.9 \mathrm{~Hz}, 1 \mathrm{H}), 3.80(\mathrm{~d}, J=17.9 \mathrm{~Hz}, 1 \mathrm{H}), 3.73-3.63(\mathrm{~m}$, $1 \mathrm{H}), 3.66(\mathrm{~s}, 3 \mathrm{H}), 1.90-0.92(\mathrm{~m}, 10 \mathrm{H}) ;{ }^{13} \mathrm{C}-\mathrm{NMR}\left(126 \mathrm{MHz}, \mathrm{CDCl}_{3}\right) \delta 169.08,166.89,154.89$, $152.76,152.24,138.60,134.35,132.66,131.95,131.69,129.39,127.70,126.26,125.47,124.88$, $122.71,118.05,117.39,69.62,52.62,52.09,49.17,32.68,32.63,25.41,24.83,24.77$; MS (qTOF) $m / z(\%) 568\left(\mathrm{M}^{+}+1,100\right), 373$ (25); HRMS (qTOF) Calcd for $\mathrm{C}_{27} \mathrm{H}_{27} \mathrm{~F}_{3} \mathrm{~N}_{3} \mathrm{O}_{7}: 562.1801$. Found: 562.1793.

Methyl N-(2-(tert-butylamino)-2-oxo-1-phenylethyl)-N-(3-nitro-2-oxo-2H-chromen-4-yl)glycinate (17o). Obtained from aldehyde 12a, amine 13e, isocyanide $\mathbf{1 4 b}$ and enol 15, as a yellow solid (140 mg, 60\%); m.p. 139-141 C; IR ( $\left.\mathrm{cm}^{-1}\right)$ 3378, 2969, 1685, 1605, 1554, 1456, 1365, 1213, 759; ${ }^{1} \mathrm{H}-\mathrm{NMR}\left(500 \mathrm{MHz}, \mathrm{CDCl}_{3}\right) \delta 8.10(\mathrm{dd}, J=8.1,1.2 \mathrm{~Hz}, 1 \mathrm{H}), 7.62(\mathrm{t}, J=7.0 \mathrm{~Hz}, 1 \mathrm{H}), 7.44-7.29$ $(\mathrm{m}, 7 \mathrm{H}), 5.84(\mathrm{bs}, 1 \mathrm{H}), 5.31(\mathrm{~s}, 1 \mathrm{H}), 4.16(\mathrm{~d}, J=18.1 \mathrm{~Hz}, 1 \mathrm{H}), 3.78(\mathrm{~d}, J=18.1 \mathrm{~Hz}, 1 \mathrm{H}), 3.65(\mathrm{~s}$, 3H), 1.25 (s, 9H); ${ }^{13} \mathrm{C}-\mathrm{NMR}\left(126 \mathrm{MHz} \mathrm{CDCl}_{3}\right) \delta 169.19$ (C), 167.67 (C), 155.18 (C), 152.70 (C), $152.65(\mathrm{C}), 134.63(\mathrm{C}), 134.04(\mathrm{CH}), 129.63(\mathrm{CH}), 129.36(\mathrm{CH}), 129.04(\mathrm{C}), 128.96(\mathrm{CH}), 128.70(\mathrm{C})$, $128.00(\mathrm{CH}), 125.24(\mathrm{CH}), 117.90(\mathrm{CH}), 70.68(\mathrm{CH}), 52.51\left(\mathrm{CH}_{3}\right), 52.21(\mathrm{C}), 51.89\left(\mathrm{CH}_{2}\right), 28.78$ $\left(\mathrm{CH}_{3}\right), 28.72\left(\mathrm{CH}_{3}\right), 28.47\left(\mathrm{CH}_{3}\right)$; MS (qTOF) $m / z(\%) 468\left(\mathrm{M}^{+}+1,15\right), 279$ (100); HRMS (qTOF) Calcd for $\mathrm{C}_{24} \mathrm{H}_{26} \mathrm{~N}_{3} \mathrm{O}_{7}$ : 468.1771. Found: 468.1751 .

Methyl N-(3-nitro-2-oxo-2H-chromen-4-yl)-N-(2-oxo-2-(pentylamino)-1-phenylethyl) glycinate (17p). Obtained from aldehyde 12a, amine 13e, isocyanide $14 \mathrm{c}$ and enol 15, as a yellow solid (92 mg, 38\%); m.p. 72-74 ${ }^{\circ} \mathrm{C}$; IR $\left(\mathrm{cm}^{-1}\right)$ 3333, 2956, 1740, 1736, 1650, 1606, 1556, 1454, 1374, 1280, 1209, 1060, 761; ${ }^{1} \mathrm{H}-\mathrm{NMR}\left(500 \mathrm{MHz}, \mathrm{CDCl}_{3}\right) \delta 8.11(\mathrm{dd}, J=8.1,1.3 \mathrm{~Hz}, 1 \mathrm{H}), 7.63$ $(\mathrm{dt}, J=6.2,1.4 \mathrm{~Hz}, 1 \mathrm{H}), 7.42-7.35(\mathrm{~m}, 7 \mathrm{H}), 5.95(\mathrm{t}, J=5.5 \mathrm{~Hz}, 1 \mathrm{H}), 5.40(\mathrm{~s}, 1 \mathrm{H}), 4.16(\mathrm{~d}, J=17.9$ $\mathrm{Hz}, 1 \mathrm{H}), 3.81(\mathrm{~d}, J=18.0 \mathrm{~Hz}, 1 \mathrm{H}), 3.65(\mathrm{~s}, 3 \mathrm{H}), 3.25-3.17(\mathrm{~m}, 2 \mathrm{H}), 1.45-1.36(\mathrm{~m}, 2 \mathrm{H}), 1.28-1.20$ $(\mathrm{m}, 2 \mathrm{H}), 1.20-1.11(\mathrm{~m}, 2 \mathrm{H}), 0.83(\mathrm{t}, J=7.2 \mathrm{~Hz}, 3 \mathrm{H}) ;{ }^{13} \mathrm{C}-\mathrm{NMR}\left(126 \mathrm{MHz}, \mathrm{CDCl}_{3}\right) \delta 169.19$ (C), $168.56(\mathrm{C}), 155.14(\mathrm{C}), 152.76(\mathrm{C}), 152.41(\mathrm{C}), 134.45(\mathrm{C}), 134.05(\mathrm{CH}), 129.75(\mathrm{CH}), 129.39$ $(\mathrm{CH}), 129.06(\mathrm{CH}), 127.88(\mathrm{CH}), 125.34(\mathrm{CH}), 117.93(\mathrm{CH}), 117.60(\mathrm{C}), 70.23(\mathrm{CH}), 52.50\left(\mathrm{CH}_{3}\right)$, $51.95\left(\mathrm{CH}_{2}\right), 40.04\left(\mathrm{CH}_{2}\right), 28.99\left(\mathrm{CH}_{2}\right), 22.34\left(\mathrm{CH}_{2}\right), 14.03\left(\mathrm{CH}_{3}\right) ; \mathrm{MS}(\mathrm{qTOF}) \mathrm{m} / z(\%) 482\left(\mathrm{M}^{+}\right.$ $+1,100), 395$ (37); HRMS (qTOF) Calcd for $\mathrm{C}_{25} \mathrm{H}_{28} \mathrm{~N}_{3} \mathrm{O}_{7}$ : 482.1927. Found: 482.1916.

Methyl N-(2-(benzylamino)-2-oxo-1-phenylethyl)-N-(3-nitro-2-oxo-2H-chromen-4-yl)glycinate (17q). Obtained from aldehyde $\mathbf{1 2 a}$, amine $\mathbf{1 3 e}$, isocyanide $\mathbf{1 4} \mathbf{d}$ and enol $\mathbf{1 5}$, as a yellow solid (118 mg, 47\%); m.p. 145-147 ${ }^{\circ} \mathrm{C}$; IR ( $\left.\mathrm{cm}^{-1}\right)$ 3296, 2946, 1744, 1722, 1651, 1602, 1556, 1532, 1454, 1410, 1215, 1179, 1054, 759, 698; ${ }^{1} \mathrm{H}-\mathrm{NMR}\left(500 \mathrm{MHz}, \mathrm{CDCl}_{3}\right) \delta 8.10(\mathrm{~d}, J=7.2 \mathrm{~Hz}, 1 \mathrm{H})$, $7.62(\mathrm{dt}, J=7.1,1.4 \mathrm{~Hz}, 1 \mathrm{H}), 7.42-7.36(\mathrm{~m}, 5 \mathrm{H}), 7.34(\mathrm{~d}, J=7.8 \mathrm{~Hz}, 2 \mathrm{H}), 7.27-7.23(\mathrm{~m}, 3 \mathrm{H})$, $7.08(\mathrm{dd}, J=6.4,3.0 \mathrm{~Hz}, 2 \mathrm{H}), 6.32(\mathrm{t}, J=5.6 \mathrm{~Hz}, 1 \mathrm{H}), 5.47(\mathrm{~s}, 1 \mathrm{H}), 4.38(\mathrm{dd}, J=5.8,1.3 \mathrm{~Hz}, 2 \mathrm{H})$, $4.14(\mathrm{~d}, J=18.0 \mathrm{~Hz}, 1 \mathrm{H}), 3.81(\mathrm{~d}, J=18.0 \mathrm{~Hz}, 1 \mathrm{H}), 3.62(\mathrm{~s}, 3 \mathrm{H}) ;{ }^{13} \mathrm{C}-\mathrm{NMR}\left(126 \mathrm{MHz}, \mathrm{CDCl}_{3}\right) \delta$ $1169.19(\mathrm{C}), 168.52(\mathrm{C}), 155.05(\mathrm{C}), 152.68(\mathrm{C}), 152.37(\mathrm{C}), 137.43(\mathrm{C}), 134.23(\mathrm{C}), 134.05(\mathrm{CH})$, $129.82(\mathrm{CH}), 129.44(\mathrm{CH}), 129.07(\mathrm{CH}), 128.87(\mathrm{CH}), 127.81(\mathrm{CH}), 125.35(\mathrm{CH}), 117.93(\mathrm{CH})$, $117.52(\mathrm{C}), 70.09(\mathrm{CH}), 52.49\left(\mathrm{CH}_{3}\right), 51.93\left(\mathrm{CH}_{2}\right), 43.97\left(\mathrm{CH}_{2}\right) ; \mathrm{MS}(\mathrm{qTOF}) \mathrm{m} / z(\%) 502\left(\mathrm{M}^{+}+\right.$ 1, 100), 415 (24), 299 (17); HRMS (qTOF) Calcd for $\mathrm{C}_{27} \mathrm{H}_{24} \mathrm{~N}_{3} \mathrm{O}_{7}$ : 502.1614. Found: 502.1602.

Methyl N-(2-(benzylamino)-2-oxo-1-(p-tolyl)ethyl)-N-(3-nitro-2-oxo-2H-chromen-4-yl)glycinate (17r). Obtained from aldehyde 12c, amine 13e, isocyanide $\mathbf{1 4 d}$ and enol 15, as a yellow solid (124 mg, 48\%); m.p. 146-148 ${ }^{\circ} \mathrm{C}$; IR ( $\left.\mathrm{cm}^{-1}\right)$ 3294, 1747, 1720, 1655, 1601, 1554, 1530, 1452, 1436, 1368, 1209, 1178, 1053, 794; ${ }^{1} \mathrm{H}-\mathrm{NMR}\left(500 \mathrm{MHz}, \mathrm{CDCl}_{3}\right) \delta 8.05(\mathrm{~d}, J=7.4 \mathrm{~Hz}, 1 \mathrm{H}), 7.62(\mathrm{t}, J=$ 
$7.3 \mathrm{~Hz}, 1 \mathrm{H}), 7.38-7.20(\mathrm{~m}, 10 \mathrm{H}), 7.14-7.11(\mathrm{~m}, 2 \mathrm{H}), 6.26(\mathrm{t}, J=5.6 \mathrm{~Hz}, 1 \mathrm{H}), 5.78(\mathrm{~s}, 1 \mathrm{H}), 4.41$ $(\mathrm{d}, J=5.9 \mathrm{~Hz}, 2 \mathrm{H}), 4.23(\mathrm{~d}, J=18.3 \mathrm{~Hz}, 1 \mathrm{H}), 3.74(\mathrm{~d}, J=18.3 \mathrm{~Hz}, 1 \mathrm{H}), 3.64(\mathrm{~s}, 3 \mathrm{H}), 2.29$ (s, 3H); ${ }^{13} \mathrm{C}-\mathrm{NMR}\left(126 \mathrm{MHz}, \mathrm{CDCl}_{3}\right) \delta 169.11$ (C), $169.04(\mathrm{C}), 155.06$ (C), 152.66 (C), 152.49 (C), $137.93(\mathrm{C}), 137.51(\mathrm{C}), 134.03(\mathrm{C}), 132.57(\mathrm{C}), 131.89(\mathrm{CH}), 129.70(\mathrm{CH}), 128.92(\mathrm{CH}), 128.48$ $(\mathrm{CH}), 127.87(\mathrm{CH}), 127.66(\mathrm{CH}), 126.9(\mathrm{CH}) 3,125.30(\mathrm{CH}), 118.09(\mathrm{CH}), 117.25(\mathrm{C}), 66.23(\mathrm{CH})$, $52.58\left(\mathrm{CH}_{3}\right), 51.45\left(\mathrm{CH}_{2}\right), 44.00\left(\mathrm{CH}_{2}\right), 19.77\left(\mathrm{CH}_{3}\right)$; MS (qTOF) $m / z(\%) 516\left(\mathrm{M}^{+}+1,17\right), 429$ (100), 299 (10); HRMS (qTOF) Calcd for $\mathrm{C}_{28} \mathrm{H}_{26} \mathrm{~N}_{3} \mathrm{O}_{7}$ : 516.1771. Found: 516.1782.

Ethyl 3-((2-(cyclohexylamino)-2-oxo-1-phenylethyl)(3-nitro-2-oxo-2H-chromen-4-yl)amino)propanoate (17s). Obtained from aldehyde 12a, amine 13f, isocyanide $\mathbf{1 4 a}$ and enol 15, as a yellow solid (131 mg, 50\%); m.p. 130-131 ${ }^{\circ} \mathrm{C}$; IR ( $\left.\mathrm{cm}^{-1}\right)$ 3367, 2931, 2854, 1732, 1682, 1603, 1553, 1451, 1200, 1052, 762; ${ }^{1} \mathrm{H}-\mathrm{NMR}\left(500 \mathrm{MHz}, \mathrm{CDCl}_{3}\right) \delta 8.05(\mathrm{dd}, J=8.1,1.2 \mathrm{~Hz}, 1 \mathrm{H}), 7.65(\mathrm{~d}, J=7,3,1.3 \mathrm{~Hz}, 1 \mathrm{H})$, $7.45-7.34(\mathrm{~m}, 7 \mathrm{H}), 5.99(\mathrm{~d}, J=8.0 \mathrm{~Hz}, 1 \mathrm{H}), 5.02(\mathrm{~s}, 1 \mathrm{H}), 4.01(\mathrm{q}, J=7.1 \mathrm{~Hz}, 2 \mathrm{H}), 3.83(\mathrm{dt}, J=14.6$, $7.2 \mathrm{~Hz}, 1 \mathrm{H}), 3.70-3.62(\mathrm{~m}, 1 \mathrm{H}), 3.43-3.36(\mathrm{~m}, 1 \mathrm{H}), 2.62(\mathrm{dt}, J=17.0,7.1 \mathrm{~Hz}, 1 \mathrm{H}), 2.44(\mathrm{dt}, J=17.1$, $6.2 \mathrm{~Hz}, 1 \mathrm{H}), 1.91(\mathrm{~d}, J=10.9 \mathrm{~Hz}, 1 \mathrm{H}), 1.75-1.52(\mathrm{~m}, 5 \mathrm{H}), 1.36-1.16(\mathrm{~m}, 3 \mathrm{H}), 1.13(\mathrm{t}, J=9.0 \mathrm{~Hz}, 3 \mathrm{H})$, 0.98 (ddd, $J=23.4,12.1,3.4 \mathrm{~Hz}, 1 \mathrm{H}) ;{ }^{13} \mathrm{C}-\mathrm{NMR}\left(126 \mathrm{MHz}, \mathrm{CDCl}_{3}\right) \delta 171.43(\mathrm{C}), 167.65(\mathrm{C}), 155.05$ (C), $153.46(\mathrm{C}), 152.85(\mathrm{C}), 134.86(\mathrm{C}), 134.03(\mathrm{CH}), 132.08(\mathrm{C}), 129.61(\mathrm{CH}), 129.32(\mathrm{CH}), 128.97$ $(\mathrm{CH}), 127.89(\mathrm{CH}), 125.25(\mathrm{CH}), 118.04(\mathrm{C}), 117.89(\mathrm{CH}), 70.02(\mathrm{CH}), 61.03\left(\mathrm{CH}_{2}\right), 49.14(\mathrm{CH}), 47.30$ $\left(\mathrm{CH}_{2}\right), 32.63\left(\mathrm{CH}_{2}\right), 32.54\left(\mathrm{CH}_{2}\right), 32.52\left(\mathrm{CH}_{2}\right), 27.05\left(\mathrm{CH}_{2}\right), 25.49\left(\mathrm{CH}_{2}\right), 24.94\left(\mathrm{CH}_{2}\right), 24.83\left(\mathrm{CH}_{2}\right)$, $14.12\left(\mathrm{CH}_{3}\right)$; MS (qTOF) $m / z(\%) 522\left(\mathrm{M}^{+}+1,100\right), 481$ (17), 333 (20); HRMS (qTOF) Calcd for $\mathrm{C}_{28} \mathrm{H}_{32} \mathrm{~N}_{3} \mathrm{O}_{7}$ : 522.2240. Found: 522.2231.

\subsubsection{General Procedure for the Reduction of Nitro Derivatives 17a-s}

To a vigorously stirred solution of enol-Ugi adduct $17 \mathbf{a}-\mathbf{s}(0.4 \mathrm{mmol})$ in glacial acetic acid $(8 \mathrm{~mL})$, iron powder $(9.6 \mathrm{mmol}, 24$ equiv) was added in one portion. The reaction mixture was stirred at $\mathrm{rt}$ for $2-4 \mathrm{~h}$. Then water $(50 \mathrm{~mL})$ and dichloromethane $(25 \mathrm{~mL})$ were added. The unreacted iron was removed by filtration and the filtrate transferred to a separatory funnel. The phases were separated, and the aqueous layer extracted again with dichloromethane $(25 \mathrm{~mL})$. The combined organic extracts were washed with water $(25 \mathrm{~mL})$, saturated $\mathrm{NaHCO}_{3}(10 \mathrm{~mL})$ and water again $(25 \mathrm{~mL})$, and then dried $\left(\mathrm{Na}_{2} \mathrm{SO}_{4}\right)$ and evaporated to dryness. The residue was purified by flash column chromatography $\left(\mathrm{SiO}_{2}\right.$, gradient from $100 \%$ hexanes to hexanes-AcOEt 7:3) to give, depending on the case, chromeno[3,4- $b$ ] piperazines $\mathbf{1 9} \mathbf{a}-\mathbf{i}, \mathbf{s}$, aminocoumarins $\mathbf{1 8} \mathbf{j}, \mathbf{k}$ or chromeno[3,4- $b]$ piperazines 201-r (Tables 1 and 2).

Synthesis and Characterization of chromeno[3,4-b]piperazines $\mathbf{1 9 a} \mathbf{a}-\mathbf{i}, \mathbf{s}$

1-Benzyl-2-phenyl-1,4-dihydro-2H-chromeno[3,4-b]pyrazine-3,5-dione (19a). Obtained from 17a as a pale yellow solid (130 mg, 85\%); m.p. $183-185{ }^{\circ} \mathrm{C}$; IR $\left(\mathrm{cm}^{-1}\right) 3254,1677,1620$, 1567, 1495, 1465, 1427, 1357, 1182, 1101, 1046, 746, 701; ${ }^{1} \mathrm{H}-\mathrm{NMR}\left(500 \mathrm{MHz}, \mathrm{CDCl}_{3}\right) \delta 7.98$ $(\mathrm{dd}, J=8.0,1.3 \mathrm{~Hz}, 1 \mathrm{H}), 7.84(\mathrm{bs}, 1 \mathrm{H}), 7.53(\mathrm{dt}, J=7.3,1.5 \mathrm{~Hz}, 1 \mathrm{H}), 7.41(\mathrm{t}, J=7.3 \mathrm{~Hz}, 2 \mathrm{H})$, 7.39-7.30 (m, 8H), 7.28-7.23 (m, 2H), $4.98(\mathrm{~s}, 1 \mathrm{H}), 4.87(\mathrm{~d}, J=15.2 \mathrm{~Hz}, 1 \mathrm{H}), 4.71(\mathrm{~d}, J=15.2$ $\mathrm{Hz}, 1 \mathrm{H}) ;{ }^{13} \mathrm{C}-\mathrm{NMR}\left(126 \mathrm{MHz}, \mathrm{CDCl}_{3}\right) \delta 163.37(\mathrm{C}), 156.63(\mathrm{C}), 150.85(\mathrm{C}), 135.88(\mathrm{C}), 135.67$ (C), $130.46(\mathrm{CH}), 129.33(\mathrm{CH}), 129.02(\mathrm{CH}), 128.75(\mathrm{CH}), 128.47(\mathrm{CH}), 127.93(\mathrm{CH}), 126.04$ $(\mathrm{CH}), 125.15(\mathrm{CH}), 123.34(\mathrm{CH}), 118.01(\mathrm{CH}), 116.55(\mathrm{C}), 112.25(\mathrm{C}), 63.91(\mathrm{CH}), 58.03\left(\mathrm{CH}_{2}\right)$; HRMS (qTOF) Calcd for $\mathrm{C}_{24} \mathrm{H}_{19} \mathrm{~N}_{2} \mathrm{O}_{3}: 383.1396$. Found: 383.1381 .

1-Benzyl-2-(2-bromophenyl)-1,4-dihydro-2H-chromeno[3,4-b]pyrazine-3,5-dione (19b). Obtained from $\mathbf{1 7 b}$ as a pale yellow solid (155 mg, 84\%); m.p. 240-242 ${ }^{\circ} \mathrm{C}$; IR (cm $\left.{ }^{-1}\right) 3195$, $3085,2938,1700,1688,1625,1495,1467,1392,1346,1094,755,699 ;{ }^{1} \mathrm{H}-\mathrm{NMR}(500 \mathrm{MHz}$, $\left.\mathrm{CDCl}_{3}\right) \delta 7.89(\mathrm{bs}, 1 \mathrm{H}), 7.74(\mathrm{dd}, J=8.0,1.1 \mathrm{~Hz}, 1 \mathrm{H}), 7.61(\mathrm{dd}, J=7.7,1.3 \mathrm{~Hz}, 1 \mathrm{H}), 7.48(\mathrm{dt}, J$ $=6.9,1.3 \mathrm{~Hz}, 1 \mathrm{H}), 7.41-7.28(\mathrm{~m}, 5 \mathrm{H}), 7.20-7.10(\mathrm{~m}, 4 \mathrm{H}), 7.04(\mathrm{dd}, J=7.5,1.8 \mathrm{~Hz}, 1 \mathrm{H}), 5.33$ $(\mathrm{s}, 1 \mathrm{H}), 5.03(\mathrm{~d}, J=14.7 \mathrm{~Hz}, 1 \mathrm{H}), 4.87(\mathrm{~d}, J=14.7 \mathrm{~Hz}, 1 \mathrm{H}) ;{ }^{13} \mathrm{C}-\mathrm{NMR}\left(126 \mathrm{MHz}, \mathrm{CDCl}_{3}\right) \delta$ 162.69 (C), 156.79 (C), $150.85(\mathrm{C}), 136.26(\mathrm{C}), 135.91(\mathrm{C}), 135.69(\mathrm{C}), 133.90(\mathrm{CH}), 130.47(\mathrm{CH})$, $130.30(\mathrm{CH}), 129.21(\mathrm{CH}), 128.91(\mathrm{CH}), 128.33(\mathrm{CH}), 128.16(\mathrm{CH}), 128.08(\mathrm{CH}), 124.93(\mathrm{CH})$, $124.53(\mathrm{C}), 124.12(\mathrm{CH}), 117.82(\mathrm{CH}), 116.47(\mathrm{C}), 111.90(\mathrm{C}), 64.07\left(\mathrm{CH}_{2}\right), 59.43(\mathrm{CH})$; HRMS (qTOF) Calcd for $\mathrm{C}_{24} \mathrm{H}_{18} \mathrm{BrN}_{2} \mathrm{O}_{3}: 461.0501$. Found: 461.0473. 
1-Benzyl-2-(p-tolyl)-1,4-dihydro-2H-chromeno[3,4-b]pyrazine-3,5-dione (19c). Obtained from 17c as a white solid (124 mg, 78\%); m.p. 170-172 ${ }^{\circ} \mathrm{C}$; IR $\left(\mathrm{cm}^{-1}\right)$ 3396, 3272, 1692, 1619, 1495, 1363, 1206, 1113, 755; ${ }^{1} \mathrm{H}-\mathrm{NMR}\left(500 \mathrm{MHz}, \mathrm{CDCl}_{3}\right) \delta 7.96(\mathrm{dd}, J=8.0,1.3 \mathrm{~Hz}, 1 \mathrm{H})$, 7.79 (bs, 1H), $7.52(\mathrm{dt}, J=7.4,1.4 \mathrm{~Hz}, 1 \mathrm{H}), 7.43-7.30(\mathrm{~m}, 7 \mathrm{H}), 7.23(\mathrm{~d}, J=8.1 \mathrm{~Hz}, 2 \mathrm{H}), 7.06$ $(\mathrm{d}, J=8.0 \mathrm{~Hz}, 2 \mathrm{H}), 4.93(\mathrm{~s}, 1 \mathrm{H}), 4.86(\mathrm{~d}, J=15.2 \mathrm{~Hz}, 1 \mathrm{H}), 4.69(\mathrm{~d}, J=15.2 \mathrm{~Hz}, 1 \mathrm{H}), 2.26(\mathrm{~s}$, 3H); ${ }^{13} \mathrm{C}-\mathrm{NMR}\left(126 \mathrm{MHz}, \mathrm{CDCl}_{3}\right) \delta 163.50$ (C), 156.63 (C), 150.86 (C), 138.32 (C), 135.97 (C), $135.86(\mathrm{C}), 132.76(\mathrm{C}), 130.38(\mathrm{CH}), 129.71(\mathrm{CH}), 129.32(\mathrm{CH}), 128.71(\mathrm{CH}), 127.95(\mathrm{CH})$, $125.99(\mathrm{CH}), 125.09(\mathrm{CH}), 123.37(\mathrm{CH}), 117.98(\mathrm{CH}), 116.65(\mathrm{C}), 112.29(\mathrm{C}), 63.79(\mathrm{CH}), 57.99$ $\left(\mathrm{CH}_{2}\right), 21.12\left(\mathrm{CH}_{3}\right)$; MS (qTOF) $m / z(\%) 397\left(\mathrm{M}^{+}+1,100\right), 337$ (30); HRMS (qTOF) Calcd for $\mathrm{C}_{25} \mathrm{H}_{21} \mathrm{~N}_{2} \mathrm{O}_{3}$ : 397.1552. Found: 397.1544.

1-Benzyl-2-(4-(trifluoromethyl)phenyl)-1,4-dihydro-2H-chromeno[3,4-b]pyrazine-3,5-dione (19d). Obtained from 17d as a pale yellow solid (160 mg, 89\%); m.p. 172-174 ${ }^{\circ} \mathrm{C}$; IR $\left(\mathrm{cm}^{-1}\right) 3438$, 3260, 1684, 1620, 1498, 1469, 1414, 1361, 1327, 1169, 1115, 1069, 752, 732; ${ }^{1} \mathrm{H}-\mathrm{NMR}(500 \mathrm{MHz}$, $\left.\mathrm{CDCl}_{3}\right) \delta 7.99(\mathrm{~d}, J=6.8 \mathrm{~Hz}, 1 \mathrm{H}), 7.84(\mathrm{bs}, 1 \mathrm{H}), 7.57-7.48(\mathrm{~m}, 5 \mathrm{H}), 7.44(\mathrm{~d}, J=8.5 \mathrm{~Hz}, 2 \mathrm{H})$, 7.39-7.30 (m, 5H), $5.02(\mathrm{~s}, 1 \mathrm{H}), 4.84(\mathrm{~d}, J=15.1 \mathrm{~Hz}, 1 \mathrm{H}), 4.70(\mathrm{~d}, J=15.1 \mathrm{~Hz}, 1 \mathrm{H}) ;{ }^{13} \mathrm{C}-\mathrm{NMR}$ $\left(126 \mathrm{MHz}, \mathrm{CDCl}_{3}\right) \delta 162.77$ (C), 156.50 (C), 150.88 (C), 139.60 (C), 135.56 (C), 135.42 (C), 130.73 $(\mathrm{CH}), 129.45(\mathrm{CH}), 128.97(\mathrm{CH}), 128.00(\mathrm{CH}), 126.53(\mathrm{CH}), 126.06(\mathrm{CH}), 126.03(\mathrm{C}), 125.38(\mathrm{CH})$, $123.11(\mathrm{CH}), 118.16(\mathrm{CH}), 116.42(\mathrm{C}), 112.72(\mathrm{C}), 63.64(\mathrm{CH}), 58.15\left(\mathrm{CH}_{2}\right) ; \mathrm{MS}(\mathrm{qTOF}) \mathrm{m} / \mathrm{z}$ (\%) $451\left(\mathrm{M}^{+}+1,95\right), 391$ (100); HRMS (qTOF) Calcd for $\mathrm{C}_{25} \mathrm{H}_{18} \mathrm{~F}_{3} \mathrm{~N}_{2} \mathrm{O}_{3}$ : 451.1270. Found: 451.1264 .

1-(Benzo[d][1,3]dioxol-5-ylmethyl)-2-phenyl-1,4-dihydro-2H-chromeno[3,4-b]pyrazine-3,5-dione (19e). Obtained from 17e as a pale yellow solid (147 mg, 86\%); m.p. 174-176 ${ }^{\circ} \mathrm{C}$; IR $\left(\mathrm{cm}^{-1}\right)$ $3264,1692,1626,1500,1445,1375,1354,1319,1253,1180,1106,1039,926,851,747,704 ;{ }^{1} \mathrm{H}-$ $\operatorname{NMR}\left(500 \mathrm{MHz}, \mathrm{CDCl}_{3}\right) \delta 7.97(\mathrm{~d}, J=4.0 \mathrm{~Hz}, 1 \mathrm{H}), 7.95(\mathrm{~d}, J=1.2 \mathrm{~Hz}, 1 \mathrm{H}), 7.53(\mathrm{dt}, J=7.0 \mathrm{~Hz}$, $1.4 \mathrm{~Hz}, 1 \mathrm{H}), 7.41(\mathrm{~d}, J=7.8 \mathrm{~Hz}, 2 \mathrm{H}), 7.36(\mathrm{dd}, J=7.0,1.5 \mathrm{~Hz}, 2 \mathrm{H}), 7.28-7.22(\mathrm{~m}, 3 \mathrm{H}), 6.80-6.75$ $(\mathrm{m}, 3 \mathrm{H}), 5.96(\mathrm{~s}, 2 \mathrm{H}), 5.00(\mathrm{~s}, 1 \mathrm{H}), 4.79(\mathrm{~d}, J=14.9 \mathrm{~Hz}, 1 \mathrm{H}), 4.59(\mathrm{~d}, J=14.9 \mathrm{~Hz}, 1 \mathrm{H}) ;{ }^{13} \mathrm{C}-\mathrm{NMR}$ $\left(126 \mathrm{MHz} \mathrm{CDCl}_{3}\right) \delta 163.44(\mathrm{C}), 156.59$ (C), 150.80 (C), 148.52 (C), $148.02(\mathrm{C}), 135.73(\mathrm{C}), 135.65$ (C), $130.41(\mathrm{CH}), 129.55(\mathrm{C}), 128.99(\mathrm{CH}), 128.42(\mathrm{CH}), 125.99(\mathrm{CH}), 125.09(\mathrm{CH}), 123.32(\mathrm{CH})$, $121.76(\mathrm{CH}), 117.98(\mathrm{CH}), 116.48(\mathrm{C}), 112.21(\mathrm{C}), 108.78(\mathrm{CH}), 108.06(\mathrm{CH}), 101.46\left(\mathrm{CH}_{2}\right), 63.46$ (CH), $57.69\left(\mathrm{CH}_{2}\right)$; MS (qTOF) $m / z(\%) 427\left(\mathrm{M}^{+}+1,100\right), 427$ (15), 274 (18); HRMS (qTOF) Calcd for $\mathrm{C}_{25} \mathrm{H}_{19} \mathrm{~N}_{2} \mathrm{O}_{5}$ : 427.1294. Found: 427.1293.

1-(Benzo[d][1,3]dioxol-5-ylmethyl)-2-(p-tolyl)-1,4-dihydro-2H-chromeno[3,4-b]pyrazine-3,5dione (19f). Obtained from $\mathbf{1 7 f}$ as a pale yellow solid (151 mg, 86\%); m.p. $213-215{ }^{\circ} \mathrm{C}$; IR $\left(\mathrm{cm}^{-1}\right) 3408,3192,1715,1677,1625,1502,1419,1326,1298,1109,1036,805,751 ;{ }^{1} \mathrm{H}-\mathrm{NMR}$ $\left(500 \mathrm{MHz}, \mathrm{CDCl}_{3}\right) \delta 7.93(\mathrm{dd}, J=8.0,1.2 \mathrm{~Hz}, 1 \mathrm{H}), 7.76(\mathrm{bs}, 1 \mathrm{H}), 7.52(\mathrm{dt}, J=7.3,1.5 \mathrm{~Hz}, 1 \mathrm{H})$, $7.43-7.36(\mathrm{~m}, 2 \mathrm{H}), 7.23(\mathrm{~d}, J=8.0 \mathrm{~Hz}, 2 \mathrm{H}), 7.06(\mathrm{~d}, J=8.0 \mathrm{~Hz}, 2 \mathrm{H}), 6.77(\mathrm{~d}, J=0.8 \mathrm{~Hz}, 2 \mathrm{H})$, $6.76(\mathrm{~s}, 1 \mathrm{H}), 5.96(\mathrm{~d}, J=0.9 \mathrm{~Hz}, 2 \mathrm{H}), 4.94(\mathrm{~s}, 1 \mathrm{H}), 4.77(\mathrm{~d}, J=14.9 \mathrm{~Hz}, 1 \mathrm{H}), 4.57(\mathrm{~d}, J=14.9 \mathrm{~Hz}$, 1H); ${ }^{13} \mathrm{C}-\mathrm{NMR}\left(126 \mathrm{MHz} \mathrm{CDCl}_{3}\right) \delta 163.52$ (C), 156.64 (C), 150.85 (C), 148.57 (C), 148.06 (C), $138.34(\mathrm{C}), 135.77(\mathrm{C}), 132.68(\mathrm{C}), 130.42(\mathrm{CH}), 129.73(\mathrm{CH}), 129.64(\mathrm{C}), 125.94(\mathrm{CH}), 125.09$ $(\mathrm{CH}), 123.38(\mathrm{CH}), 121.78(\mathrm{CH}), 118.02(\mathrm{CH}), 116.57(\mathrm{C}), 112.16(\mathrm{C}), 108.82(\mathrm{CH}), 108.08$ $(\mathrm{CH}), 101.50\left(\mathrm{CH}_{2}\right), 63.35(\mathrm{CH}), 57.69\left(\mathrm{CH}_{2}\right), 21.14\left(\mathrm{CH}_{3}\right)$; $\mathrm{MS}(\mathrm{qTOF}) \mathrm{m} / z(\%) 441\left(\mathrm{M}^{+}+1\right.$, 100), 400 (20), 281(30); HRMS (qTOF) Calcd for $\mathrm{C}_{26} \mathrm{H}_{21} \mathrm{~N}_{2} \mathrm{O}_{5}: 441.4630$. Found: 441.1449.

1-(Benzo[d][1,3]dioxol-5-ylmethyl)-2-(4-(trifluoromethyl)phenyl)-1,4-dihydro-2H-chromeno[3,4b]pyrazine-3,5-dione (19g). Obtained from $\mathbf{1 7 g}$ as a pale yellow solid (148 mg, 75\%); m.p. 190-192 ${ }^{\circ} \mathrm{C}$; IR $\left(\mathrm{cm}^{-1}\right)$ 3080, 2918, 1685, 1619, 1493, 1412, 1329, 1241, 1114, 1068, 1041, 998, 756; ${ }^{1} \mathrm{H}-\mathrm{NMR}$ $\left(500 \mathrm{MHz}, \mathrm{CDCl}_{3}\right) \delta 7.96(\mathrm{dd}, J=6.8,1.6 \mathrm{~Hz}, 1 \mathrm{H}), 7.81(\mathrm{bs}, 2 \mathrm{H}), 7.58-7.48(\mathrm{~m}, 5 \mathrm{H}), 7.46-7.41(\mathrm{~m}$, $2 \mathrm{H}), 6.80-6.75(\mathrm{~m}, 3 \mathrm{H}), 5.97(\mathrm{dd}, J=2.5,1.3 \mathrm{~Hz}, 2 \mathrm{H}), 5.03(\mathrm{~s}, 1 \mathrm{H}), 4.75(\mathrm{~d}, J=14.8 \mathrm{~Hz}, 1 \mathrm{H}), 4.58$ $(\mathrm{d}, J=14.8 \mathrm{~Hz}, 1 \mathrm{H}) ;{ }^{13} \mathrm{C}-\mathrm{NMR}\left(126 \mathrm{MHz} \mathrm{CDCl}_{3}\right) \delta 162.80(\mathrm{C}), 156.49(\mathrm{C}), 150.87$ (C), 148.71 (C), $148.29(\mathrm{C}), 139.61(\mathrm{C}), 135.30(\mathrm{C}), 130.72(\mathrm{CH}), 129.22(\mathrm{C}), 126.51(\mathrm{CH}), 126.07(\mathrm{CH}), 126.04$ $(\mathrm{CH}), 125.35(\mathrm{CH}), 123.11(\mathrm{CH}), 121.91(\mathrm{CH}), 118.18(\mathrm{CH}), 116.38(\mathrm{C}), 112.69(\mathrm{C}), 108.92(\mathrm{CH})$, $108.10(\mathrm{CH}), 101.59\left(\mathrm{CH}_{2}\right), 63.28(\mathrm{CH}), 57.89\left(\mathrm{CH}_{2}\right)$; MS (qTOF) $m / z(\%) 495\left(\mathrm{M}^{+}+1,100\right), 339$ (30), 353 (65); HRMS (qTOF) Calcd for $\mathrm{C}_{26} \mathrm{H}_{18} \mathrm{~F}_{3} \mathrm{~N}_{2} \mathrm{O}_{5}$ : 495.1168. Found: 495.1157.

1-Cyclohexyl-2-(p-tolyl)-1,4-dihydro-2H-chromeno[3,4-b]pyrazine-3,5-dione (19h). Obtained from $17 \mathrm{~h}$ as a pale yellow solid $(107 \mathrm{mg}, 69 \%)$; m.p. $249-251^{\circ} \mathrm{C}$; IR $\left(\mathrm{cm}^{-1}\right) 3444,2931,2853$, 
1692, 1622, 1495, 1406, 1335, 1102, 999, 757; ${ }^{1} \mathrm{H}-\mathrm{NMR}\left(500 \mathrm{MHz}, \mathrm{CDCl}_{3}\right) \delta 7.90$ (bs, 1H), 7.74 $(\mathrm{dd}, J=7.9,1.4 \mathrm{~Hz}, 1 \mathrm{H}), 7.49(\mathrm{dt}, J=6.0,1.5 \mathrm{~Hz}, 1 \mathrm{H}), 7.42-7.36(\mathrm{~m}, 2 \mathrm{H}), 7.28(\mathrm{~d}, J=8.0 \mathrm{~Hz}$, 2H), $7.05(\mathrm{~d}, J=8.0 \mathrm{~Hz}, 2 \mathrm{H}), 5.17(\mathrm{~s}, 1 \mathrm{H}), 3.77(\mathrm{tt}, J=12.0,3.7 \mathrm{~Hz}, 1 \mathrm{H}), 2.31(\mathrm{~d}, J=13.3 \mathrm{~Hz}$, $1 \mathrm{H}), 2.25(\mathrm{~s}, 3 \mathrm{H}), 1.95(\mathrm{~d}, J=13.2 \mathrm{~Hz}, 1 \mathrm{H}), 1.83-1.09(\mathrm{~m}, 8 \mathrm{H}) ;{ }^{13} \mathrm{C}-\mathrm{NMR}\left(126 \mathrm{MHz}, \mathrm{CDCl}_{3}\right) \delta$ $164.43(\mathrm{C}), 156.66(\mathrm{C}), 150.87(\mathrm{C}), 138.08(\mathrm{C}), 136.15(\mathrm{CH}), 133.12(\mathrm{C}), 130.15(\mathrm{C}), 129.60(\mathrm{CH})$, $125.84(\mathrm{CH}), 124.98(\mathrm{CH}), 123.36(\mathrm{CH}), 117.92(\mathrm{CH}), 117.05(\mathrm{C}), 112.33(\mathrm{C}), 62.44(\mathrm{CH}), 58.98$ $(\mathrm{CH}), 31.92\left(\mathrm{CH}_{3}\right), 31.76\left(\mathrm{CH}_{3}\right), 26.07\left(\mathrm{CH}_{3}\right), 25.98\left(\mathrm{CH}_{3}\right), 25.24\left(\mathrm{CH}_{3}\right), 21.10\left(\mathrm{CH}_{3}\right) ; \mathrm{MS}$ (qTOF) $m / z(\%) 389\left(\mathrm{M}^{+}+1,100\right), 348$ (31), 255 (29); HRMS (qTOF) Calcd for $\mathrm{C}_{24} \mathrm{H}_{25} \mathrm{~N}_{2} \mathrm{O}_{3}$ : 389.1865. Found: 389.1866.

1-Cyclohexyl-2-(4-(trifluoromethyl)phenyl)-1,4-dihydro-2H-chromeno[3,4-b]pyrazine-3,5-dione (19i). Obtained from 17i as a pale yellow solid (127 mg, 72\%); m.p. $113-115{ }^{\circ} \mathrm{C}$; IR $\left(\mathrm{cm}^{-1}\right)$ 3427, 2932, 2856, 1692, 1622, 1411, 1326, 1165, 1125, 1069, 755; ${ }^{1} \mathrm{H}-\mathrm{NMR}\left(500 \mathrm{MHz}, \mathrm{CDCl}_{3}\right)$ $\delta 8.00(\mathrm{bs}, 1 \mathrm{H}), 7.75(\mathrm{dd}, J=7.9,1.2 \mathrm{~Hz}, 1 \mathrm{H}), 7.57-7.50(\mathrm{~m}, 6 \mathrm{H}), 7.45-7.36(\mathrm{~m}, 2 \mathrm{H}), 5.24(\mathrm{~s}$, $1 \mathrm{H}), 3.79(\mathrm{tt}, J=12.0,3.6 \mathrm{~Hz}, 1 \mathrm{H}), 2.30(\mathrm{~d}, J=12.6 \mathrm{~Hz}, 1 \mathrm{H}), 1.96(\mathrm{~d}, J=13.1 \mathrm{~Hz}, 1 \mathrm{H}), 1.85-1.58$ (m, 5H), 1.29-1.11 (m, 3H); ${ }^{13} \mathrm{C}-\mathrm{NMR}\left(126 \mathrm{MHz}, \mathrm{CDCl}_{3}\right) \delta 163.73(\mathrm{C}), 156.52(\mathrm{C}), 150.91$ (C), $140.21(\mathrm{C}), 135.73(\mathrm{C}), 130.46(\mathrm{CH}), 126.42(\mathrm{CH}), 125.96(\mathrm{CH}), 125.93(\mathrm{CH}), 125.20(\mathrm{CH})$, $123.11(\mathrm{CH}), 118.10(\mathrm{CH}), 116.80(\mathrm{C}), 112.54(\mathrm{C}), 62.62(\mathrm{CH}), 59.06(\mathrm{CH}), 31.86\left(\mathrm{CH}_{2}\right), 31.73$ $\left(\mathrm{CH}_{2}\right), 27.07\left(\mathrm{CH}_{2}\right), 25.95\left(\mathrm{CH}_{2}\right), 25.21\left(\mathrm{CH}_{2}\right)$; MS (qTOF) $m / z(\%) 443\left(\mathrm{M}^{+}+1,100\right), 301(<5)$; HRMS (qTOF) Calcd for $\mathrm{C}_{24} \mathrm{H}_{22} \mathrm{~F}_{3} \mathrm{~N}_{2} \mathrm{O}_{3}: 443.1583$. Found: 443.1578 .

Ethyl 3-(3,5-dioxo-2-phenyl-3,4-dihydro-2H-chromeno[3,4-b]pyrazin-1(5H)-yl)propanoate (19s). Obtained from 17s as a white solid (133 mg, 85\%); m.p. $145-147^{\circ} \mathrm{C}$; IR (cm $\left.{ }^{-1}\right) 3231$, 1733, 1680, 1619, 1498, 1460, 1372, 1188, 1126, 1021, 757; ${ }^{1} \mathrm{H}-\mathrm{NMR}\left(500 \mathrm{MHz}, \mathrm{CDCl}_{3}\right) \delta 7.99$ $(\mathrm{s}, 1 \mathrm{H}), 7.89(\mathrm{dd}, J=7.9,1.1 \mathrm{~Hz}, 1 \mathrm{H}), 7.52(\mathrm{dt}, J=7.3,1.2 \mathrm{~Hz}, 1 \mathrm{H}), 7.43-7.36(\mathrm{~m}, 4 \mathrm{H}), 7.29-7.24$ $(\mathrm{m}, 3 \mathrm{H}), 5.09(\mathrm{~s}, 1 \mathrm{H}), 4.17-4.06(\mathrm{~m}, 3 \mathrm{H}), 3.89(\mathrm{ddd}, J=14.7,8.2,6.4 \mathrm{~Hz}, 1 \mathrm{H}), 2.79(\mathrm{ddd}, J=$ $14.6,8.1,6.4 \mathrm{~Hz}, 1 \mathrm{H}), 2.73-2.63(\mathrm{~m}, 1 \mathrm{H}), 1.19(\mathrm{t}, J=7.1 \mathrm{~Hz}, 3 \mathrm{H}) ;{ }^{13} \mathrm{C}-\mathrm{NMR}\left(126 \mathrm{MHz}, \mathrm{CDCl}_{3}\right)$ $\delta 170.71$ (C), 163.61 (C), 156.53 (C), 150.72 (C), 135.51 (C), 135.15 (C), 130.45 (CH), 129.06 $(\mathrm{CH}), 128.57(\mathrm{CH}), 125.81(\mathrm{CH}), 125.15(\mathrm{CH}), 123.39(\mathrm{CH}), 117.98(\mathrm{CH}), 116.43(\mathrm{C}), 112.23$ (C), $64.73(\mathrm{CH}), 61.31\left(\mathrm{CH}_{2}\right), 50.18\left(\mathrm{CH}_{2}\right), 33.96\left(\mathrm{CH}_{2}\right), 14.15\left(\mathrm{CH}_{3}\right) ; \mathrm{HRMS}(\mathrm{qTOF})$ Calcd for $\mathrm{C}_{22} \mathrm{H}_{21} \mathrm{~N}_{2} \mathrm{O}_{5}$ : 393.1450. Found: 393.1444.

Synthesis and Characterization of Aminocoumarins $\mathbf{1 8 j}, \mathbf{k}$

2-((3-Amino-2-oxo-2H-chromen-4-yl)(phenyl)amino)-N-cyclohexyl-2-phenylacetamide (18j). Obtained from 17j as a white solid (99 mg,53\%); m.p. 203-205 ${ }^{\circ} \mathrm{C}$; IR ( $\left.\mathrm{cm}^{-1}\right) 3466,2933$, 2852, 1719, 1635, 1600, 1556, 1497, 1455, 1177, 746; ${ }^{1} \mathrm{H}-\mathrm{NMR}\left(500 \mathrm{MHz}, \mathrm{CDCl}_{3}\right) \delta 7.27-7.18$ $(\mathrm{m}, 4 \mathrm{H}), 7.11-7.05(\mathrm{~m}, 3 \mathrm{H}), 7.04-6.96(\mathrm{~m}, 4 \mathrm{H}), 6.83(\mathrm{t}, J=7.3 \mathrm{~Hz}, 1 \mathrm{H}), 6.64(\mathrm{~d}, J=8.1 \mathrm{~Hz}$, $2 \mathrm{H}), 6.21(\mathrm{bs}, 2 \mathrm{H}), 5.65(\mathrm{~d}, J=8.1 \mathrm{~Hz}, 1 \mathrm{H}), 5.51(\mathrm{~s}, 1 \mathrm{H}), 3.93-3.83(\mathrm{~m}, 1 \mathrm{H}), 2.15-0.92(\mathrm{~m}$, 10H); ${ }^{13} \mathrm{C}-\mathrm{NMR}\left(126 \mathrm{MHz}, \mathrm{CDCl}_{3}\right) \delta 171.49$ (C), 160.18 (C), 147.29 (C), 145.30 (C), 134.85 (C), $132.81(\mathrm{C}), 129.70(\mathrm{CH}), 129.48(\mathrm{CH}), 129.42(\mathrm{CH}), 128.12(\mathrm{CH}), 125.71(\mathrm{CH}), 123.94(\mathrm{CH})$, $122.65(\mathrm{CH}), 121.88(\mathrm{C}), 119.66(\mathrm{C}), 118.93(\mathrm{CH}), 115.93(\mathrm{CH}), 112.72(\mathrm{CH}), 68.52(\mathrm{CH}), 49.44$ $(\mathrm{CH}), 33.19\left(\mathrm{CH}_{2}\right), 32.69\left(\mathrm{CH}_{2}\right), 25.55\left(\mathrm{CH}_{2}\right), 24.98\left(\mathrm{CH}_{2}\right), 24.84\left(\mathrm{CH}_{2}\right) ; \mathrm{MS}(\mathrm{qTOF}) \mathrm{m} / z(\%)$ $468\left(\mathrm{M}^{+}+1,5,407\right.$ (18), 369 (100); HRMS (qTOF) Calcd for $\mathrm{C}_{29} \mathrm{H}_{30} \mathrm{~N}_{3} \mathrm{O}_{3}$ : 468.2287. Found: 468.2285.

2-((3-Amino-2-oxo-2H-chromen-4-yl)(phenyl)amino)-N-cyclohexyl-2-(3,4-dimethoxyphenyl) acetamide (18k). Obtained from 17k as a white solid (163 mg, 77\%); m.p. 243-245 ${ }^{\circ} \mathrm{C}$; IR $\left(\mathrm{cm}^{-1}\right) 3439,3326,2938,2850,1715,1637,1599,1539,1518,1464,1253,1176,1152,1028,763$, 749; ${ }^{1} \mathrm{H}-\mathrm{NMR}\left(500 \mathrm{MHz}, \mathrm{CDCl}_{3}\right) \delta 7.20(\mathrm{t}, J=7.9 \mathrm{~Hz}, 2 \mathrm{H}), 7.11-7.06(\mathrm{~m}, 2 \mathrm{H}), 6.98(\mathrm{~d}, J=3.5$ $\mathrm{Hz}, 2 \mathrm{H}), 6.82-6.78(\mathrm{~m}, 2 \mathrm{H}), 6.74(\mathrm{~s}, 1 \mathrm{H}), 6.61(\mathrm{~d}, J=8.2 \mathrm{~Hz}, 2 \mathrm{H}), 6.50(\mathrm{~d}, J=8.3 \mathrm{~Hz}, 1 \mathrm{H}), 6.20$ (bs, 2H), $5.60(\mathrm{~d}, J=8.1 \mathrm{~Hz}, 1 \mathrm{H}), 5.44(\mathrm{~s}, 1 \mathrm{H}), 3.90-3.80(\mathrm{~m}, 1 \mathrm{H}), 3.71(\mathrm{~s}, 3 \mathrm{H}), 3.52(\mathrm{~s}, 3 \mathrm{H})$, 2.08-0.79 (m, 10H); ${ }^{13} \mathrm{C}-\mathrm{NMR}\left(126 \mathrm{MHz} \mathrm{CDCl}_{3}\right) \delta 171.68(\mathrm{C}), 160.15(\mathrm{C}), 149.69(\mathrm{C}), 148.24$ (C), $147.43(\mathrm{C}), 145.24(\mathrm{C}), 134.75(\mathrm{C}), 129.71(\mathrm{CH}), 125.75(\mathrm{CH}), 124.96(\mathrm{CH}), 123.95(\mathrm{CH})$, $122.57(\mathrm{CH}), 121.99(\mathrm{C}), 119.86(\mathrm{CH}), 118.89(\mathrm{CH}), 116.26(\mathrm{CH}), 112.65(\mathrm{CH}), 110.32(\mathrm{CH})$, $68.09(\mathrm{CH}), 55.87\left(\mathrm{CH}_{3}\right), 55.79\left(\mathrm{CH}_{3}\right), 49.43(\mathrm{CH}), 33.21\left(\mathrm{CH}_{2}\right), 32.77\left(\mathrm{CH}_{2}\right), 29.84\left(\mathrm{CH}_{2}\right)$, $24.98\left(\mathrm{CH}_{2}\right), 24.86\left(\mathrm{CH}_{2}\right)$; MS (qTOF) $m / z(\%) 528\left(\mathrm{M}^{+}+1,100\right), 369$ (25), 276 (13); HRMS (qTOF) Calcd for $\mathrm{C}_{31} \mathrm{H}_{34} \mathrm{~N}_{3} \mathrm{O}_{5}: 528.2498$. Found: 528.2490. 
Synthesis and Characterization of chromeno[3,4-b]piperazines 201-r

$\mathrm{N}$-cyclohexyl-2-(3,5-dioxo-3,4-dihydro-2H-chromeno[3,4-b]pyrazin-1(5H)-yl)-2-phenyl acetamide (201). Obtained from 171 as a pale yellow solid (121 mg, 70\%); m.p. $240-242{ }^{\circ} \mathrm{C}$; IR $\left(\mathrm{cm}^{-1}\right) 3268,2931,2854,1703,1818,1544,1496,1451,1382,1111,752 ;{ }^{1} \mathrm{H}-\mathrm{NMR}(500 \mathrm{MHz}$, $\left.\mathrm{CDCl}_{3}\right) \delta 7.63(\mathrm{dd}, J=8.0,1.2 \mathrm{~Hz}, 1 \mathrm{H}), 7.54(\mathrm{bs}, 1 \mathrm{H}), 7.51(\mathrm{dt}, J=7.1,1.4 \mathrm{~Hz}, 1 \mathrm{H}), 7.43-7.39$ $(\mathrm{m}, 4 \mathrm{H}), 7.34-7.29(\mathrm{~m}, 3 \mathrm{H}), 5.79(\mathrm{~d}, J=8.0 \mathrm{~Hz}, 1 \mathrm{H}), 5.37(\mathrm{~s}, 1 \mathrm{H}), 3.98-3.90(\mathrm{~m}, 1 \mathrm{H}), 3.92(\mathrm{~s}$, $3 \mathrm{H}), 2.03-1.90(\mathrm{~m}, 2 \mathrm{H}), 1.74-1.57(\mathrm{~m}, 4 \mathrm{H}), 1.45-1.07\left(\mathrm{~m}, 4 \mathrm{H} . ;{ }^{13} \mathrm{C}-\mathrm{NMR}\left(126 \mathrm{MHz}, \mathrm{CDCl}_{3}\right) \delta\right.$ $167.85(\mathrm{C}), 163.00(\mathrm{C}), 156.92(\mathrm{C}), 150.63(\mathrm{C}), 135.72(\mathrm{C}), 135.01(\mathrm{C}), 130.32(\mathrm{CH}), 129.52(\mathrm{CH})$, $128.50(\mathrm{CH}), 125.06(\mathrm{CH}), 122.96(\mathrm{CH}), 118.02(\mathrm{CH}), 116.33(\mathrm{C}), 114.08(\mathrm{C}), 69.14(\mathrm{CH}), 49.49$ $\left(\mathrm{CH}_{2}\right), 49.05(\mathrm{CH}), 33.17\left(\mathrm{CH}_{2}\right), 33.10\left(\mathrm{CH}_{2}\right), 25.51\left(\mathrm{CH}_{2}\right), 24.89\left(\mathrm{CH}_{2}\right), 24.85\left(\mathrm{CH}_{2}\right) ; \mathrm{MS}$ (qTOF) $m / z(\%) 432\left(\mathrm{M}^{+}+1,100\right), 305(<5), 234(<5)$; HRMS (qTOF) Calcd for $\mathrm{C}_{25} \mathrm{H}_{26} \mathrm{~N}_{3} \mathrm{O}_{4}$ : 432.1923. Found: 432.1914.

$\mathrm{N}$-cyclohexyl-2-(3,5-dioxo-3,4-dihydro-2H-chromeno[3,4-b]pyrazin-1(5H)-yl)-2-(p-tolyl) acetamide (20m). Obtained from $17 \mathrm{~m}$ as a pale yellow solid (98 $\mathrm{mg}, 55 \%)$; m.p. $245-247^{\circ} \mathrm{C}$ (dec.); IR ( $\mathrm{cm}^{-1}$ ) 3400, 3280, 2930, 2849, 1701, 1651, 1618, 1561, 1439, 1412, 1347, 1108, 751, 732; ${ }^{1} \mathrm{H}-\mathrm{NMR}\left(500 \mathrm{MHz}, \mathrm{CDCl}_{3}\right) \delta 7.67$ (bs, $\left.1 \mathrm{H}\right), 7.47(\mathrm{t}, J=7.2 \mathrm{~Hz}, 1 \mathrm{H}), 7.44-7.37(\mathrm{~m}, 3 \mathrm{H})$, 7.35-7.31 (m, 2H), 7.26-7.19 (m, 2H), $5.72(\mathrm{~d}, J=8.1 \mathrm{~Hz}, 1 \mathrm{H}), 5.59(\mathrm{~s}, 1 \mathrm{H}), 4.09(\mathrm{~d}, J=16.7 \mathrm{~Hz}$, $1 \mathrm{H}), 3.98(\mathrm{~d}, J=16.7 \mathrm{~Hz}, 1 \mathrm{H}), 3.95-3.87(\mathrm{~m}, 1 \mathrm{H}), 1.98-0.95(\mathrm{~m}, 10 \mathrm{H}) ;{ }^{13} \mathrm{C}-\mathrm{NMR}(126 \mathrm{MHz}$, $\left.\mathrm{CDCl}_{3}\right) \delta 168.26(\mathrm{C}), 162.61(\mathrm{C}), 156.91(\mathrm{C}), 150.61(\mathrm{C}), 137.43(\mathrm{C}), 136.82(\mathrm{C}), 133.84(\mathrm{C})$, $131.87(\mathrm{CH}), 130.36(\mathrm{CH}), 129.58(\mathrm{CH}), 128.28(\mathrm{CH}), 127.23(\mathrm{CH}), 124.88(\mathrm{CH}), 123.08(\mathrm{CH})$, $118.05(\mathrm{CH}), 116.39(\mathrm{C}), 111.84(\mathrm{C}), 67.33(\mathrm{CH}), 49.97(\mathrm{CH}), 49.08\left(\mathrm{CH}_{2}\right), 33.00\left(\mathrm{CH}_{2}\right), 25.47$ $\left(\mathrm{CH}_{2}\right), 24.81\left(\mathrm{CH}_{2}\right), 19.44\left(\mathrm{CH}_{3}\right)$; MS (qTOF) $\mathrm{m} / \mathrm{z}(\%) 446\left(\mathrm{M}^{+}+1,11\right), 319$ (100); HRMS (qTOF) Calcd for $\mathrm{C}_{26} \mathrm{H}_{28} \mathrm{~N}_{3} \mathrm{O}_{4}$ : 446.2080. Found: 446.2074 .

N-cyclohexyl-2-(3,5-dioxo-3,4-dihydro-2H-chromeno[3,4-b]pyrazin-1(5H)-yl)-2-(4-(trifluoromethyl) phenyl)acetamide (20n). Obtained from $17 \mathrm{n}$ as a pale yellow solid (162 mg, 81\%); m.p. $249-251^{\circ} \mathrm{C}$ (dec.); $\mathbb{I R}\left(\mathrm{cm}^{-1}\right)$ 3313, 3266, 2930, 2855, 1704, 1688, 1620, 1549, 1497, 1487, 1326, 1167, 1126, 1068, $824,{ }^{1} \mathrm{H}-\mathrm{NMR}\left(500 \mathrm{MHz}, \mathrm{CDCl}_{3}\right) \delta 7.68(\mathrm{~d}, J=8.2 \mathrm{~Hz}, 1 \mathrm{H}), 7.60(\mathrm{bs}, 1 \mathrm{H}), 7.58(\mathrm{dd}, J=8.1,1.2 \mathrm{~Hz}$, $1 \mathrm{H}), 7.53(\mathrm{t}, J=7.8 \mathrm{~Hz}, 1 \mathrm{H}), 7.45-7.34(\mathrm{~m}, 3 \mathrm{H}), 7.39-7.34(\mathrm{~m}, 1 \mathrm{H}), 5.88(\mathrm{~d}, J=4.7 \mathrm{~Hz}, 1 \mathrm{H}), 5.37(\mathrm{~s}$, $1 \mathrm{H}), 3.98-3.88(\mathrm{~m}, 1 \mathrm{H}), 3.91(\mathrm{~d}, J=2.9 \mathrm{~Hz}, 2 \mathrm{H}), 2.04-1.09(\mathrm{~m}, 10 \mathrm{H}) ;{ }^{13} \mathrm{C}-\mathrm{NMR}\left(126 \mathrm{MHz}, \mathrm{CDCl}_{3}\right)$ $\delta 167.11(\mathrm{C}), 162.84(\mathrm{C}), 156.76(\mathrm{C}), 150.56(\mathrm{C}), 138.72(\mathrm{C}), 134.97(\mathrm{C}), 130.56(\mathrm{CH}), 128.98(\mathrm{CH})$, $127.12(\mathrm{C}), 126.43(\mathrm{CH}), 125.26(\mathrm{CH}), 122.53(\mathrm{CH}), 118.18(\mathrm{CH}), 116.04(\mathrm{C}), 114.70(\mathrm{C}), 68.39(\mathrm{CH})$, $49.41\left(\mathrm{CH}_{2}\right), 49.20(\mathrm{CH}), 33.18\left(\mathrm{CH}_{2}\right), 33.11\left(\mathrm{CH}_{2}\right), 25.44\left(\mathrm{CH}_{2}\right), 24.88\left(\mathrm{CH}_{2}\right) ; \mathrm{MS}(\mathrm{qTOF}) \mathrm{m} / \mathrm{z}$ (\%) $522\left(\mathrm{M}^{+}+\mathrm{Na}^{+}, 100\right), 500\left(\mathrm{M}^{+}+1,54\right), 429$ (10); HRMS (qTOF) Calcd for $\mathrm{C}_{26} \mathrm{H}_{25} \mathrm{~F}_{3} \mathrm{~N}_{3} \mathrm{O}_{4}$ : 500.1797. Found: 500.1782.

$\mathrm{N}$-(tert-butyl)-2-(3,5-dioxo-3,4-dihydro-2H-chromeno[3,4-b]pyrazin-1(5H)-yl)-2-phenylacetamide (20o). Obtained from $17 \mathrm{o}$ as a pale yellow solid $(94 \mathrm{mg}, 58 \%)$; m.p. $258-260{ }^{\circ} \mathrm{C}\left(\mathrm{dec}\right.$ ); $\mathrm{IR}\left(\mathrm{cm}^{-1}\right)$ 3328, 3269, 2966, 2931, 1680, 1619, 1561, 1496, 1466, 1365, 1288, 1111, 750; ${ }^{1} \mathrm{H}-\mathrm{NMR}(500 \mathrm{MHz}$, $\left.\mathrm{CDCl}_{3}\right) \delta .59(\mathrm{dd}, J=8.0,1.2 \mathrm{~Hz}, 1 \mathrm{H}), 7.53-7.49(\mathrm{~m}, 2 \mathrm{H}), 7.44-7.40(\mathrm{~m}, 4 \mathrm{H}), 7.34-7.29(\mathrm{~m}, 3 \mathrm{H})$, $5.71(\mathrm{bs}, 1 \mathrm{H}), 5.31(\mathrm{~s}, 1 \mathrm{H}), 3.96(\mathrm{q}, J=16.9 \mathrm{~Hz}, 2 \mathrm{H}), 1.41(\mathrm{~s}, 9 \mathrm{H}) ;{ }^{13} \mathrm{C}-\mathrm{NMR}\left(126 \mathrm{MHz}, \mathrm{CDCl}_{3}\right) \delta$ $168.11(\mathrm{C}), 163.00(\mathrm{C}), 156.94(\mathrm{C}), 150.63(\mathrm{C}), 135.98(\mathrm{C}), 135.19(\mathrm{C}), 130.34(\mathrm{CH}), 129.54(\mathrm{CH})$, $129.50(\mathrm{CH}), 128.40(\mathrm{CH}), 125.04(\mathrm{CH}), 123.00(\mathrm{CH}), 118.01(\mathrm{CH}), 116.32(\mathrm{C}), 113.75(\mathrm{C}), 69.53$ $(\mathrm{CH}), 52.50(\mathrm{C}), 49.54\left(\mathrm{CH}_{2}\right), 28.85\left(\mathrm{CH}_{3}\right)$; MS (qTOF) $\mathrm{m} / z(\%) 406\left(\mathrm{M}^{+}+1,60\right), 321(27), 279$ (100); HRMS (qTOF) Calcd for $\mathrm{C}_{23} \mathrm{H}_{24} \mathrm{~N}_{3} \mathrm{O}_{4}$ : 406.1767. Found: 406.1761.

2-(3,5-Dioxo-3,4-dihydro-2H-chromeno[3,4-b]pyrazin-1(5H)-yl)-N-pentyl-2-phenylacetamide (20p). Obtained from $17 \mathrm{p}$ as a white solid $(92 \mathrm{mg}, 55 \%)$; m.p. $206-208{ }^{\circ} \mathrm{C}$ (dec.); IR ( $\left.\mathrm{cm}^{-1}\right)$ 3310, 3276, 2929, 1687, 1616, 1567, 1496, 1471, 1380, 1115, 999, 931, 748, 726; ${ }^{1}$ H-NMR (500 $\left.\mathrm{MHz}, \mathrm{CDCl}_{3}\right) \delta 7.63(\mathrm{dd}, J=8.0,1.2 \mathrm{~Hz}, 1 \mathrm{H}), 7.51(\mathrm{dt}, 6.9, J=1.3 \mathrm{~Hz}, 2 \mathrm{H}), 7.43-7.37(\mathrm{~m}, 4 \mathrm{H})$, 7.35-7.28 (m, 4H), $6.04(\mathrm{t}, J=5.4 \mathrm{~Hz}, 1 \mathrm{H}), 5.39(\mathrm{~s}, 1 \mathrm{H}), 3.92(\mathrm{~d}, J=1.7 \mathrm{~Hz}, 2 \mathrm{H}), 3.37(\mathrm{dd}, J=$ $13.2,7.0 \mathrm{~Hz}, 2 \mathrm{H}), 1.57-1.50(\mathrm{~m}, 2 \mathrm{H}), 1.37-1.25(\mathrm{~m}, 4 \mathrm{H}), 0.90(\mathrm{t}, J=7.1 \mathrm{~Hz}, 3 \mathrm{H}) ;{ }^{13} \mathrm{C}-\mathrm{NMR}$ $\left(126 \mathrm{MHz}, \mathrm{CDCl}_{3}\right) \delta 168.65(\mathrm{C}), 162.80$ (C), 156.78 (C), 150.47 (C), 135.51 (C), 134.77 (C), $130.21(\mathrm{CH}), 129.44(\mathrm{CH}), 129.39(\mathrm{CH}), 128.38(\mathrm{CH}), 124.94(\mathrm{CH}), 122.83(\mathrm{CH}), 117.87(\mathrm{CH})$, $116.16(\mathrm{C}), 114.07(\mathrm{C}), 69.04(\mathrm{CH}), 49.40\left(\mathrm{CH}_{2}\right), 40.00\left(\mathrm{CH}_{2}\right), 29.19\left(\mathrm{CH}_{2}\right), 29.04\left(\mathrm{CH}_{2}\right), 22.27$ $\left(\mathrm{CH}_{2}\right), 13.97\left(\mathrm{CH}_{3}\right)$; MS (qTOF) $m / z(\%) 420\left(\mathrm{M}^{+}+1,14\right), 293$ (100); HRMS (qTOF) Calcd for $\mathrm{C}_{24} \mathrm{H}_{26} \mathrm{~N}_{3} \mathrm{O}_{4}$ : 420.1923. Found: 420.1918 . 
N-benzyl-2-(3,5-dioxo-3,4-dihydro-2H-chromeno[3,4-b]pyrazin-1(5H)-yl)-2-phenylacetamide (20q). Obtained from 17q as a white solid (118 mg, 67\%); m.p. $238-240{ }^{\circ} \mathrm{C}$ (dec.); IR (cm ${ }^{-1}$ ) 3280, 1686, 1618, 1496, 1379, 1113, 751, 700; ${ }^{1} \mathrm{H}-\mathrm{NMR}\left(500 \mathrm{MHz}, \mathrm{CDCl}_{3}\right) \delta 7.59(\mathrm{dd}, J=8.0$, $1.1 \mathrm{~Hz}, 1 \mathrm{H}), 7.52-7.44(\mathrm{~m}, 2 \mathrm{H}), 7.42-7.33(\mathrm{~m}, 6 \mathrm{H}), 7.33-7.25(\mathrm{~m}, 6 \mathrm{H}), 6.33(\mathrm{t}, J=5.5 \mathrm{~Hz}, 1 \mathrm{H})$, $5.42(\mathrm{~s}, 1 \mathrm{H}), 4.56(\mathrm{qd}, J=14.6,5.8 \mathrm{~Hz}, 2 \mathrm{H}), 3.92(d, \mathrm{~J}=1.7 \mathrm{~Hz}, 2 \mathrm{H}) ;{ }^{13} \mathrm{C}-\mathrm{NMR}(126 \mathrm{MHz}$, $\left.\mathrm{CDCl}_{3}\right) \delta 168.90(\mathrm{C}), 162.97(\mathrm{C}), 156.87(\mathrm{C}), 150.57(\mathrm{C}), 137.66(\mathrm{C}), 135.55(\mathrm{C}), 134.61(\mathrm{C})$, $130.39(\mathrm{CH}), 129.63(\mathrm{CH}), 129.52(\mathrm{CH}), 129.06(\mathrm{CH}), 128.55(\mathrm{CH}), 128.06(\mathrm{CH}), 128.01(\mathrm{CH})$, $125.10(\mathrm{CH}), 122.89(\mathrm{CH}), 118.00(\mathrm{CH}), 116.18(\mathrm{C}), 114.29(\mathrm{C}), 69.11(\mathrm{CH}), 49.43\left(\mathrm{CH}_{2}\right), 44.15$ $\left(\mathrm{CH}_{2}\right)$; MS (qTOF) $m / z(\%) 440\left(\mathrm{M}^{+}+1,100\right), 282$ (40), 169 (15); HRMS (qTOF) Calcd for $\mathrm{C}_{26} \mathrm{H}_{22} \mathrm{~N}_{3} \mathrm{O}_{4}$ : 440.1610. Found: 440.1585 .

N-benzyl-2-(3,5-dioxo-3,4-dihydro-2H-chromeno[3,4-b]pyrazin-1(5H)-yl)-2-(p-tolyl)acetamide (20r). Obtained from 17r as a white solid (141 mg, 78\%); m.p. $221-223^{\circ} \mathrm{C}$ (dec.); IR (cm ${ }^{-1}$ ) $3369,1711,1665,1649,1559,1495,1113,754,703 ;{ }^{1} \mathrm{H}-\mathrm{NMR}\left(500 \mathrm{MHz}, \mathrm{CDCl}_{3}\right) \delta 7.79$ (bs, 1H), $7.58(\mathrm{dt}, J=7.2,1.2 \mathrm{~Hz}, 1 \mathrm{H}), 7.54(\mathrm{dd}, J=8.1,1.2 \mathrm{~Hz}, 1 \mathrm{H}), 7.51-7.54(\mathrm{~m}, 5 \mathrm{H}), 7.42-7.38(\mathrm{~m}, 5 \mathrm{H})$, $7.36-7.23(\mathrm{~m}, 2 \mathrm{H}), 6.38(\mathrm{t}, J=6.4 \mathrm{~Hz}, 1 \mathrm{H}), 5.79(\mathrm{~s}, 1 \mathrm{H}), 4.75(\mathrm{dd}, J=14.6,6.2 \mathrm{~Hz}, 1 \mathrm{H}), 4.57(\mathrm{dd}$, $J=14.6,5.5 \mathrm{~Hz}, 1 \mathrm{H}), 4.21(\mathrm{~d}, J=16.7 \mathrm{~Hz}, 1 \mathrm{H}), 4.09(\mathrm{~d}, J=16.7 \mathrm{~Hz}, 1 \mathrm{H}), 2.14(\mathrm{~s}, 3 \mathrm{H}) ;{ }^{13} \mathrm{C}-\mathrm{NMR}$ $\left(126 \mathrm{MHz}, \mathrm{CDCl}_{3}\right) \delta 169.46(\mathrm{C}), 162.69$ (C), 156.85 (C), 150.57 (C), 137.65 (C), 137.27 (C), 136.89 (C), $133.46(\mathrm{C}), 131.91(\mathrm{CH}), 130.43(\mathrm{CH}), 129.69(\mathrm{CH}), 129.06(\mathrm{CH}), 128.65(\mathrm{CH}), 128.12(\mathrm{CH})$, $128.06(\mathrm{CH}), 127.18(\mathrm{CH}), 124.95(\mathrm{CH}), 123.05(\mathrm{CH}), 118.07(\mathrm{CH}), 116.26(\mathrm{C}), 112.10(\mathrm{C}), 66.93$ $(\mathrm{CH}), 49.79\left(\mathrm{CH}_{2}\right), 44.24\left(\mathrm{CH}_{2}\right), 19.44\left(\mathrm{CH}_{3}\right)$; MS (qTOF) $m / z(\%) 454\left(\mathrm{M}^{+}+1,27\right), 369(73)$, 327 (100); HRMS (qTOF) Calcd for $\mathrm{C}_{27} \mathrm{H}_{24} \mathrm{~N}_{3} \mathrm{O}_{4}$ : 454.1767. Found: 454.1761.

\subsubsection{General Procedure for the Synthesis of Chromeno[3,4-b]piperazines 19j,k}

Enol-Ugi adduct 17j-k $(0.4 \mathrm{mmol})$ and iron powder $(9.6 \mathrm{mmol}, 24$ equiv $)$ in glacial acetic acid $(8 \mathrm{~mL})$, were subjected to a procedure identical to the one used for the reduction of nitro derivatives $\mathbf{1 7 a}-\mathbf{s}$, except that the reaction was performed at $150{ }^{\circ} \mathrm{C}$. Chromeno[3,4$b$ ]piperazines $\mathbf{1 9} \mathbf{j}, \mathbf{k}$ were obtained after flash column chromatography purification $\left(\mathrm{SiO}_{2}\right.$, gradient from $100 \%$ hexanes to hexanes-AcOEt, 7:3).

1,2-Diphenyl-1,4-dihydro-2H-chromeno[3,4-b]pyrazine-3,5-dione (19j). Obtained from 18j as a pale yellow solid (103 mg, 70\%); m.p. 243-245 ${ }^{\circ} \mathrm{C}$; IR $\left(\mathrm{cm}^{-1}\right) 3423,3075,1716,1679$, 1631, 1492, 1470, 1407, 1369, 1234, 1131, 998, 754, 593; ${ }^{1} \mathrm{H}-\mathrm{NMR}\left(500 \mathrm{MHz}, \mathrm{CDCl}_{3}\right) \delta 8.15$ (bs, $1 \mathrm{H}), 7.51(\mathrm{dd}, J=6.3,1.7 \mathrm{~Hz}, 2 \mathrm{H}), 7.43-7.30(\mathrm{~m}, 7 \mathrm{H}), 7.22(\mathrm{t}, J=7.4 \mathrm{~Hz}, 1 \mathrm{H}), 7.15(\mathrm{dd}, J=$ 8.1, 1.2 Hz, 1H), $7.09(\mathrm{~d}, J=8.0 \mathrm{~Hz}, 2 \mathrm{H}), 7.06(\mathrm{~d}, J=7.3 \mathrm{~Hz}, 1 \mathrm{H}), 5.64(\mathrm{~s}, 1 \mathrm{H}) ;{ }^{13} \mathrm{C}-\mathrm{NMR}(126$ $\left.\mathrm{MHz} \mathrm{CDCl}_{3}\right) \delta 162.88(\mathrm{C}), 156.74(\mathrm{C}), 150.88(\mathrm{C}), 145.76(\mathrm{C}), 135.81(\mathrm{C}), 131.77(\mathrm{C}), 130.07$ $(\mathrm{CH}), 130.00(\mathrm{CH}), 129.41(\mathrm{CH}), 128.86(\mathrm{CH}), 125.89(\mathrm{CH}), 125.63(\mathrm{CH}), 124.99(\mathrm{CH}), 124.40$ (CH), $122.53(\mathrm{CH}), 117.80(\mathrm{CH}), 115.63(\mathrm{C}), 111.31(\mathrm{C}), 69.02(\mathrm{CH}) ; \mathrm{MS}(\mathrm{qTOF}) \mathrm{m} / z(\%) 369$ $\left(\mathrm{M}^{+}+1,100\right), 288(<5)$; HRMS (qTOF) Calcd for $\mathrm{C}_{23} \mathrm{H}_{17} \mathrm{~N}_{2} \mathrm{O}_{3}: 369.1239$. Found: 369.1228.

2-(3,4-Dimethoxyphenyl)-1-phenyl-1,4-dihydro-2H-chromeno[3,4-b]pyrazine-3,5-dione (19k). Obtained from 18k as a pale yellow solid (120 mg, 70\%); m.p. 209-211 ${ }^{\circ} \mathrm{C}$; IR $\left(\mathrm{cm}^{-1}\right) 3423$, 3072, 1712, 1678, 1632, 1604, 1514, 1403, 1260, 1140, 1024, 999, 757; ${ }^{1} \mathrm{H}-\mathrm{NMR}(500 \mathrm{MHz}$, $\left.\mathrm{CDCl}_{3}\right) \delta 8.12(\mathrm{bs}, 1 \mathrm{H}), 7.44-7.31(\mathrm{~m}, 4 \mathrm{H}), 7.22(\mathrm{t}, J=7.4 \mathrm{~Hz}, 1 \mathrm{H}), 7.15(\mathrm{dd}, J=8.1,1.1 \mathrm{~Hz}$, $1 \mathrm{H}), 7.12-7.04(\mathrm{~m}, 4 \mathrm{H}), 6.98(\mathrm{dd}, J=8.4,2.1 \mathrm{~Hz}, 1 \mathrm{H}), 6.79(\mathrm{~d}, J=8.4 \mathrm{~Hz}, 1 \mathrm{H}), 5.59(\mathrm{~s}, 1 \mathrm{H})$, 3.83 (s, 3H), 3.72 (s, 3H); ${ }^{13} \mathrm{C}-\mathrm{NMR}\left(126 \mathrm{MHz} \mathrm{CDCl}_{3}\right) \delta 163.24$ (C), 156.70 (C), 150.79 (C), $149.64(\mathrm{C}), 149.40(\mathrm{C}), 145.48(\mathrm{C}), 131.68(\mathrm{C}), 130.10(\mathrm{CH}), 129.97(\mathrm{CH}), 127.65(\mathrm{C}), 125.76$ $(\mathrm{CH}), 124.73(\mathrm{CH}), 124.35(\mathrm{CH}), 122.25(\mathrm{CH}), 117.86(\mathrm{CH}), 117.44(\mathrm{CH}), 115.53(\mathrm{C}), 111.63$ $(\mathrm{CH}), 111.28(\mathrm{C}), 108.67(\mathrm{CH}), 68.31(\mathrm{CH}), 56.07\left(\mathrm{CH}_{3}\right), 55.91\left(\mathrm{CH}_{3}\right) ; \mathrm{MS}(\mathrm{qTOF}) \mathrm{m} / z(\%) 429$ $\left(\mathrm{M}^{+}+1,75\right), 369$ (100), 276 (45); HRMS (qTOF) Calcd for $\mathrm{C}_{25} \mathrm{H}_{21} \mathrm{~N}_{2} \mathrm{O}_{5}$ : 429.1450. Found: 429.1454 .

\section{Conclusions}

Multicomponent functionalization of 4-hydroxy-3-nitro-coumarin by an enol-Ugi condensation permits to introduce a peptidic chain that is subsequently cyclized in reducing conditions to build the fused piperazino ring. In this way, rigid polyheterocyclic di- and tri-peptides comprising a wide chemical diversity are easily accessible. This strategy, 
involving the first reported post-condensation transformation of an enol-Ugi adduct, opens new opportunities for the discovery of novel pharmacologically active compounds.

Supplementary Materials: The following are available online: Figure S1: Aldehydes, amines, and isocyanides used as starting materials, Figure S2: Imines used as starting materials, Experimental data for imine $16 \mathrm{~g}$, Copies of the NMR spectra for all new compounds.

Author Contributions: Conceptualization, A.G.N. and C.F.M.; methodology, A.B. and A.G.N.; writing-original draft preparation, C.F.M. All authors have read and agreed to the published version of the manuscript.

Funding: This research was funded by Junta de Extremadura and FEDER (IB16095).

Institutional Review Board Statement: Not applicable.

Informed Consent Statement: Not applicable.

Data Availability Statement: The data presented in this study is available in this article and in the supplementary material.

Acknowledgments: COMPUTAEX for granting access to supercomputer LUSITANIA.

Conflicts of Interest: The authors declare no conflict of interest.

Sample Availability: Samples of the compounds are available from the authors.

\section{References}

1. Giannis, A.; Kolter, T. Peptidomimetics for Receptor Ligands-Discovery, Development, and Medical Perspectives. Angew. Chem. Int. Ed. 1993, 32, 1244-1267. [CrossRef]

2. Gante, J. Peptidomimetics-Tailored Enzyme-Inhibitors. Angew. Chem. Int. Ed. 1994, 33, 1699-1720. [CrossRef]

3. Hanessian, S.; McNaughton-Smith, G.; Lombart, H.-G.; Lubell, W.D. Design and synthesis of conformationally constrained amino acids as versatile scaffolds and peptide mimetics. Tetrahedron 1997, 53, 12789-12854. [CrossRef]

4. Gopalakrishnan, R.; Frolov, A.I.; Knerr, L.; Drury, W.J., 3rd; Valeur, E. Therapeutic Potential of Foldamers: From Chemical Biology Tools To Drug Candidates? J. Med. Chem. 2016, 59, 9599-9621. [CrossRef] [PubMed]

5. Usmanova, L.; Dar'in, D.; Novikov, M.S.; Gureev, M.; Krasavin, M. Bicyclic Piperazine Mimetics of the Peptide beta-Turn Assembled via the Castagnoli-Cushman Reaction. J. Org. Chem. 2018, 83, 5859-5868. [CrossRef]

6. Limbach, M.; Lygin, A.V.; Korotkov, V.S.; Es-Sayed, M.; de Meijere, A. Facile synthesis of structurally diverse 5-oxopiperazine-2carboxylates as dipeptide mimics and templates. Org. Biomol. Chem. 2009, 7, 3338-3342. [CrossRef]

7. Golebiowski, A.; Jozwik, J.; Klopfenstein, S.R.; Colson, A.-O.; Grieb, A.L.; Russell, A.F.; Rastogi, V.L.; Diven, C.F.; Portlock, D.E.; Chen, J.J. Solid-Supported Synthesis of Putative Peptide $\beta$-Turn Mimetics via Ugi Reaction for Diketopiperazine Formation. J. Comb. Chem. 2002, 4, 584-590. [CrossRef] [PubMed]

8. Kim, H.-O.; Nakanishi, H.; Lee, M.S.; Kahn, M. Design and Synthesis of Novel Conformationally Restricted Peptide Secondary Structure Mimetics. Org. Lett. 2000, 2, 301-302. [CrossRef]

9. Golebiowski, A.; Klopfenstein, S.R.; Shao, X.; Chen, J.J.; Colson, A.-O.; Grieb, A.L.; Russell, A.F. Solid-Supported Synthesis of a Peptide $\beta$-Turn Mimetic. Org. Lett. 2000, 2, 2615-2617. [CrossRef]

10. Belov, V.N.; Funke, C.; Labahn, T.; Es-Sayed, M.; de Meijere, A. An Easy Access to Bicyclic Peptides with an Octahydro[2H]pyrazino[1,2a]pyrazine Skeleton. Eur. J. Org. Chem. 1999, 2, 1345-1356. [CrossRef]

11. Gigant, N.; Claveau, E.; Bouyssou, P.; Gillaizeau, I. Diversity-oriented synthesis of polycyclic diazinic scaffolds. Org. Lett. 2012, 14, 844-847. [CrossRef]

12. Zhao, J.; Zhao, H.; Hall, J.A.; Brown, D.; Brandes, E.; Bazzill, J.; Grogan, P.T.; Subramanian, C.; Vielhauer, G.; Cohen, M.S.; et al. Triazole Containing Novobiocin and Biphenyl Amides as Hsp90 C-Terminal Inhibitors. Medchemcomm 2014, 5, 1317-1323. [CrossRef] [PubMed]

13. Heide, L. New aminocoumarin antibiotics as gyrase inhibitors. Int. J. Med. Microbiol. 2014, 304, 31-36. [CrossRef]

14. Gunaherath, G.M.K.B.; Marron, M.T.; Wijeratne, E.M.K.; Whitesell, L.; Gunatilaka, A.A.L. Synthesis and biological evaluation of novobiocin analogues as potential heat shock protein 90 inhibitors. Biorg. Med. Chem. 2013, 21, 5118-5129. [CrossRef] [PubMed]

15. Cele, F.N.; Kumalo, H.; Soliman, M.E. Mechanism of Inhibition of Hsp90 Dimerization by Gyrase B Inhibitor Coumermycin A1 (C-A1) Revealed by Molecular Dynamics Simulations and Thermodynamic Calculations. Cell Biochem. Biophys. 2016, 74, 353-363. [CrossRef] [PubMed]

16. Zettler, J.; Xia, H.; Burkard, N.; Kulik, A.; Grond, S.; Heide, L.; Apel, A.K. New aminocoumarins from the rare actinomycete Catenulispora acidiphila DSM 44928: Identification, structure elucidation, and heterologous production. Chembiochem 2014, 15, 612-621. [CrossRef] [PubMed]

17. Jans, P.E.; Mfuh, A.M.; Arman, H.D.; Shaffer, C.V.; Larionov, O.V.; Mooberry, S.L. Cytotoxicity and Mechanism of Action of the Marine-Derived Fungal Metabolite Trichodermamide B and Synthetic Analogues. J. Nat. Prod. 2017, 80, 676-683. [CrossRef] 
18. Doens, D.; Valiente, P.A.; Mfuh, A.M.; Vo, A.X.T.; Tristan, A.; Carreno, L.; Quijada, M.; Nguyen, V.T.; Perry, G.; Larionov, O.V.; et al. Identification of Inhibitors of CD36-Amyloid Beta Binding as Potential Agents for Alzheimer's Disease. ACS Chem. Neurosci. 2017, 8, 1232-1241. [CrossRef]

19. Sun, J.; Pei, Y.; Li, E.; Li, W.; Hyde, K.D.; Yin, W.B.; Liu, X. A new species of Trichoderma hypoxylon harbours abundant secondary metabolites. Sci. Rep. 2016, 6, 37369. [CrossRef]

20. Illgen, K.; Nerdinger, S.; Fuchs, T.; Friedrich, C.; Weber, L.; Herdtweck, E. A Versatile Synthesis of 6-Oxo-1,4,5,6-tetrahydropyrazine-2-carboxylic Acid Methyl Esters via MCR Chemistry. Synlett 2004, 1, 53-56. [CrossRef]

21. Faggi, C.; Garcia-Valverde, M.; Marcaccini, S.; Pepino, R.; Pozo, M.C. Studies on Isocyanides and Related Compounds: A Facile Synthesis of 1,6-Dihydro-6-Oxopyrazine-2-Carboxylic Acid Derivatives Via Ugi Four-Component Condensation. Synthesis 2003, 10, 1553-1558. [CrossRef]

22. Jovanović, M.; Zhukovsky, D.; Podolski-Renić, A.; Domračeva, I.; Žalubovskis, R.; Senćanski, M.; Glišić, S.; Sharoyko, V.; Tennikova, T.; Dar'In, D.; et al. Novel electrophilic amides amenable by the Ugi reaction perturb thioredoxin system via thioredoxin reductase 1 (TrxR1) inhibition: Identification of DVD-445 as a new lead compound for anticancer therapy. Eur. J. Med. Chem. 2019, 181, 111580. [CrossRef] [PubMed]

23. Hulme, C.; Ma, L.; Cherrier, M.P.; Romano, J.J.; Morton, G.; Duquenne, C.; Salvino, J.; Labaudiniere, R. Novel applications of convertible isonitriles for the synthesis of mono and bicyclic gamma-lactams via a UDC strategy. Tetrahedron Lett. 2000, 41, 1883-1887. [CrossRef]

24. El Kaïm, L.; Oble, J.; Gizzi, M.; Grimaud, L. Ugi-Smiles Access to Quinoxaline Derivatives. Heterocycles 2007, 73. [CrossRef]

25. Iordanidou, D.; Zarganes-Tzitzikas, T.; Neochoritis, C.G.; Domling, A.; Lykakis, I.N. Application of Silver Nanoparticles in the Multicomponent Reaction Domain: A Combined Catalytic Reduction Methodology to Efficiently Access Potential Hypertension or Inflammation Inhibitors. ACS Omega 2018, 3, 16005-16013. [CrossRef] [PubMed]

26. Ligia, S.d.S.P.; Mara, R.C.C.; Marcus, V.N.d.S. Multicomponent Reactions in the Synthesis of Complex Fused Coumarin Derivatives. Curr. Org. Synth. 2018, 15, 21-37. [CrossRef]

27. Huang, Y.; Khoury, K.; Dömling, A. The Piperazine Space in Isocyanide-based MCR Chemistry. In Synthesis of Heterocycles via Multicomponent Reactions I; Springer Science \& Business Media: Berlin, Germany, 2010; pp. 85-127.

28. Medina, F.G.; Marrero, J.G.; Macias-Alonso, M.; Gonzalez, M.C.; Cordova-Guerrero, I.; Teissier Garcia, A.G.; Osegueda-Robles, S. Coumarin heterocyclic derivatives: Chemical synthesis and biological activity. Nat. Prod. Rep. 2015, 32, 1472-1507. [CrossRef]

29. Domling, A.; Khoury, K. Praziquantel and schistosomiasis. ChemMedChem 2010, 5, 1420-1434. [CrossRef]

30. Demetri, G.D.; Chawla, S.P.; von Mehren, M.; Ritch, P.; Baker, L.H.; Blay, J.Y.; Hande, K.R.; Keohan, M.L.; Samuels, B.L.; Schuetze, S.; et al. Efficacy and safety of trabectedin in patients with advanced or metastatic liposarcoma or leiomyosarcoma after failure of prior anthracyclines and ifosfamide: Results of a randomized phase II study of two different schedules. J. Clin. Oncol. 2009, 27, 4188-4196. [CrossRef]

31. Croom, K.F.; Perry, C.M.; Plosker, G.L. Mirtazapine: A review of its use in major depression and other psychiatric disorders. CNS Drugs 2009, 23, 427-452. [CrossRef] [PubMed]

32. Baker, M.E.; Lathe, R. The promiscuous estrogen receptor: Evolution of physiological estrogens and response to phytochemicals and endocrine disruptors. J. Steroid. Biochem. Mol. Biol. 2018, 184, 29-37. [CrossRef] [PubMed]

33. Ceci, C.; Lacal, P.M.; Tentori, L.; De Martino, M.G.; Miano, R.; Graziani, G. Experimental Evidence of the Antitumor, Antimetastatic and Antiangiogenic Activity of Ellagic Acid. Nutrients 2018, 10, 1756. [CrossRef] [PubMed]

34. François, G.; Timperman, G.; Holenz, J.; Assi, L.A.; Geuder, T.; Maes, L.; Dubois, J.; Hanocq, M.; Bringmannj, G. Naphthylisoquinoline alkaloids exhibit strong growth-inhibiting activities against Plasmodium falciparum and P. berghei in vitro-Structureactivity relationships of dioncophylline C. Ann. Trop. Med. Parasit. 2016, 90, 115-123. [CrossRef]

35. Khanolkar, A.D.; Lu, D.; Ibrahim, M.; Duclos, R.I., Jr.; Thakur, G.A.; Malan, T.P., Jr.; Porreca, F.; Veerappan, V.; Tian, X.; George, C.; et al. Cannabilactones: A novel class of CB2 selective agonists with peripheral analgesic activity. J. Med. Chem. 2007, 50, 6493-6500. [CrossRef]

36. Lin, W.; Huang, J.; Liao, X.; Yuan, Z.; Feng, S.; Xie, Y.; Ma, W. Neo-tanshinlactone selectively inhibits the proliferation of estrogen receptor positive breast cancer cells through transcriptional down-regulation of estrogen receptor alpha. Pharmacol. Res. 2016, 111, 849-858. [CrossRef] [PubMed]

37. Ostrowska, K. Coumarin-piperazine derivatives as biologically active compounds. Saudi Pharm. J. 2020, 28, 220-232. [CrossRef]

38. Kotharkar, S.A.; Shinde, D.B. Synthesis of antimicrobial 2,9,10-trisubstituted-6-oxo-7,12-dihydro-chromeno[3,4-b]quinoxalines. Bioorg. Med. Chem. Lett. 2006, 16, 6181-6184. [CrossRef]

39. Hamama, W.S.; Hassanien, A.E.; El-Fedawy, M.G.; Zoorob, H.H. Synthesis and Antimicrobial Evaluation of Novel Polyfused Heterocycles-Based Quinolone. J. Heterocycl. Chem. 2015, 52, 492-496. [CrossRef]

40. Han, G.S.; Yook, D.J.; Kim, S.K.; Shim, S.C.; Kang, H.K. Photophysical properties of pyrazinopsoralen, a new monofunctional psoralen. Photochem. Photobiol. 1996, 64, 525-530. [CrossRef]

41. Hu, C.; Zhang, Z.; Gao, W.; Zhang, G.; Liu, T.; Liu, Q. PIFA-promoted intramolecular oxidative C(aryl)-H amidation reaction: Synthesis of quinolino[3,4-b]quinoxalin-6(5 H )-ones. Tetrahedron 2018, 74, 665-671. [CrossRef]

42. Li, Y.; Lei, J.; Xu, J.; Tang, D.-Y.; Chen, Z.-Z.; Zhu, J.; Xu, C. A facile method for building fused quinoxaline-quinolinones via an acidless post-Ugi cascade reaction. Chin. Chem. Lett. 2017, 28, 541-545. [CrossRef] 
43. Shiva Kumar, K.; Rambabu, D.; Prasad, B.; Mujahid, M.; Krishna, G.R.; Basaveswara Rao, M.V.; Malla Reddy, C.; Vanaja, G.R.; Kalle, A.M.; Pal, M. A new approach to construct a fused 2-ylidene chromene ring: Highly regioselective synthesis of novel chromeno quinoxalines. Org. Biomol. Chem. 2012, 10, 4774-4781. [CrossRef] [PubMed]

44. Hoplamaz, E.; Keskin, S.; Balci, M. Regioselective Synthesis of Benzo[h][1,6]-naphthyridines and Chromenopyrazinones through Alkyne Cyclization. Eur. J. Org. Chem. 2017, 2017, 1489-1497. [CrossRef]

45. Neo, A.G.; López-García, L.; Marcos, C.F. Allylic amination of Passerini adducts. Application to the selective synthesis of chromone-substituted $\alpha$-and $\gamma$-amino acid peptidic and retropeptidic units. RSC Adv. 2014, 4, 40044-40053. [CrossRef]

46. Neo, A.; Carrillo, R.; Delgado, J.; Marcaccini, S.; Marcos, C.F. A multicomponent approach to the synthesis of 1,3-dicarbonylic compounds. Mol. Divers. 2011, 15, 529-539. [CrossRef] [PubMed]

47. Carrillo, R.M.; Neo, A.G.; Lopez-Garcia, L.; Marcaccini, S.; Marcos, C.F. Zinc catalysed ester solvolysis. Application to the synthesis of tartronic acid derivatives. Green Chem. 2006, 8, 787-789. [CrossRef]

48. Neo, A.G.; Delgado, J.; Polo, C.; Marcaccini, S.; Marcos, C.F. A new synthesis of beta-keto amides by reduction of Passerini adducts. Tetrahedron Lett. 2005, 46, 23-26. [CrossRef]

49. Castellano, T.G.; Neo, A.G.; Marcaccini, S.; Marcos, C.F. Enols as feasible Acid components in the ugi condensation. Org. Lett. 2012, 14, 6218-6221. [CrossRef]

50. Neo, A.G.; Castellano, T.G.; Marcos, C.F. An easy synthesis of diversely functionalized 2 H-chromenes and amido amines by an enol-Ugi reaction. Arkivoc 2017, 21-31. [CrossRef]

51. Neo, A.G.; Castellano, T.G.; Marcos, C.F. Enol-Ugi Reaction of Hydroxycoumarins: Straightforward Synthesis of Amino Acid Derived Coumarin Enamines. Synthesis 2015, 47, 2431-2438. [CrossRef]

52. Savel'ev, V.L.; Artamonova, O.S.; Troitskaya, V.S.; Vinokurov, V.G.; Zagorevskii, V.A. Investigations of pyrans and related compounds. Chem. Heterocycl. Compd. 1973, 9, 816-820. [CrossRef]

53. Schiff, H. Mittheilungen aus dem Universitätslaboratorium in Pisa: Eine neue Reihe organischer Basen. Justus Liebigs Ann. Chem. 1864, 131, 118-119. [CrossRef]

54. Qin, W.; Long, S.; Panunzio, M.; Biondi, S. Schiff bases: A short survey on an evergreen chemistry tool. Molecules 2013, 18, 12264-12289. [CrossRef] [PubMed] 